Article

\title{
Distance and Similarity Measures for Spherical Fuzzy Sets and Their Applications in Selecting Mega Projects
}

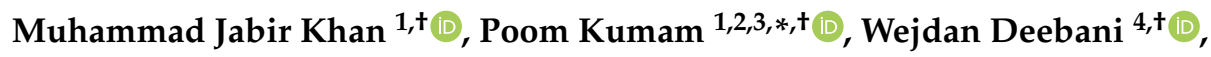 \\ Wiyada Kumam $^{5, *,+}$ (D) and Zahir Shah ${ }^{2,+}$ (D) \\ 1 KMUTT Fixed Point Research Laboratory, SCL 802 Fixed Point Laboratory and Department of Mathematics, \\ Faculty of Science, King Mongkut's University of Technology Thonburi (KMUTT), 126 Pracha-Uthit Road, \\ Bang Mod, Thrung Khru, Bangkok 10140, Thailand; jabirkhan.uos@gmail.com \\ 2 Center of Excellence in Theoretical and Computational Science (TaCS-CoE), Science Laboratory Building, \\ Faculty of Science, King Mongkut's University of Technology Thonburi (KMUTT), 126 Pracha-Uthit Road, \\ Bang Mod, Thrung Khru, Bangkok 10140, Thailand; Zahir.sha@kmutt.ac.th \\ 3 Department of Medical Research, China Medical University Hospital, China Medical University, \\ Taichung 40402, Taiwan \\ 4 Deparments of Mathematics, College of Science and Arts, King Abdulaziz University, P.O. Box 344, \\ Rabigh 21911, Saudi Arabia; wdeebani@kau.edu.sa \\ 5 Program in Applied Statistics, Department of Mathematics and Computer Science, Faculty of Science and \\ Technology, Rajamangala University of Technology Thanyaburi (RMUTT), Thanyaburi, \\ Pathumthani 12110, Thailand \\ * Correspondence: poom.kumam@mail.kmutt.ac.th (P.K.); wiyada.kum@rmutt.ac.th (W.K.); \\ Tel.: +662-470-8994 (P.K.) \\ + These authors contributed equally to this work.
}

Received: 4 March 2020; Accepted: 25 March 2020; Published: 3 April 2020

\begin{abstract}
A new condition on positive membership, neutral membership, and negative membership functions give us the successful extension of picture fuzzy set and Pythagorean fuzzy set and called spherical fuzzy sets $(\mathcal{S F S})$. This extends the domain of positive membership, neutral membership, and negative membership functions. Keeping in mind the importance of similarity measure and application in data mining, medical diagnosis, decision making, and pattern recognition, several studies on similarity measures have been proposed in the literature. Some of those, however, cannot satisfy the axioms of similarity and provide counter-intuitive cases. In this paper, we proposed the set-theoretic similarity and distance measures. We provide some counterexamples for already proposed similarity measures in the literature and shows that how our proposed method is important and applicable to the pattern recognition problems. In the end, we provide an application of a proposed similarity measure for selecting mega projects in under developed countries.
\end{abstract}

Keywords: spherical fuzzy set; distance measure; similarity measures; pattern recognition; mega projects

\section{Introduction}

The membership function is used to define the fuzzy set $(\mathcal{F S})$. The uncertainty model effectively by the fuzzy set theory define by Zadeh [1]. The fuzzy set theory only focuses on one aspect of information, the containment or belongingness. Attansove defines the intuitionistic fuzzy set $(\mathcal{I F S})$ [2], which is the generalization of FS and model uncertainty effectively. The membership and non-membership functions are used to define $\mathcal{I F S}$. Due to the consideration of non-membership function, the $\mathcal{I} \mathcal{F} \mathcal{S}$ is more effective than $\mathcal{F S}$ for practical applications. The membership functions 
for the interval fuzzy set $(\mathcal{I} v-\mathcal{F S})$ and interval-valued intuitionistic fuzzy set $(\mathcal{I} v-\mathcal{I} \mathcal{F} \mathcal{S})$ describe in intervals instead of single values [3]. The experts give their preferences in the form of intervals in $\mathcal{I}_{v}-\mathcal{F} \mathcal{S}_{s}$ and $\mathcal{I} v-\mathcal{I} \mathcal{F} \mathcal{S}_{s}$. Due to intensive quantity and type of uncertainties, these approaches are not sufficient to cover all aspects [4]. Molodtsov soft set theory model uncertainty by parametric point of view [4]. Nowadays, many authors define the hybrid model of soft sets with $\mathcal{F} \mathcal{S} s, \mathcal{I} \mathcal{F} S$ s, $\mathcal{I} v-\mathcal{F} \mathcal{S}$, and $\mathcal{I} v-\mathcal{I} \mathcal{F} \mathcal{S}_{S}[5-9]$.

The picture fuzzy set $(\mathcal{P F S})$ define by Coung is another generalization of $\mathcal{F} \mathcal{S} s$ and $\mathcal{I} \mathcal{F} \mathcal{S}_{s}$ [10]. The generalization in the sense that the membership, neutral and non-membership functions are used to define $\mathcal{P} \mathcal{F} \mathcal{S}$. In $\mathcal{P} \mathcal{F} \mathcal{S}$, the preferences of the experts describe precisely because it contains all aspects of assessment of information like yes, abstain, no and refusal. The addition of the representative's functions in $\mathcal{P} \mathcal{F} \mathcal{S}$ should be less than or equal to one. This condition restricts the expert preferences domain. The hybrid model of $\mathcal{P} \mathcal{F S}$ and soft set is obtained by Yang [11]. Khan et al. [12] define the generalized picture fuzzy soft set and applied them to decision-making problems. For study more about decision making, we refer to [13-19].

Yager $[20,21]$ defines the Pythagorean fuzzy sets $\mathcal{P} y \mathcal{F} \mathcal{S}$, which is the successful extension of intuitionistic fuzzy sets, by putting a new condition on positive membership $\xi$ and negative membership functions $v$, i.e., $0 \leq \xi^{2}+v^{2} \leq 1$. This new condition expand the domain of membership functions like if we have $\xi=0.7$ and $v=0.5$, then we cannot deal it with intuitionistic fuzzy set because $0.7+0.5 \geq 1$ but $0.7^{2}+0.5^{2}=0.49+0.25=0.74 \leq 1$ and hence $\mathcal{P} y \mathcal{F} \mathcal{S}$ applied successfully. The concept of Pythagorean fuzzy number $\mathcal{P} y \mathcal{F} \mathcal{S}$ and detailed mathematical expression of $\mathcal{P} y \mathcal{F} \mathcal{S}$ is presented by Zhang [22]. To solve the multi criteria group decision-making problem with $\mathcal{P} y \mathcal{F} \mathcal{S}$, Peng defines the division and subtraction operations for $\mathcal{P} y \mathcal{F} \mathcal{S}$ and also developed a Pythagorean fuzzy superiority and inferiority ranking method [23]. Reformat and Yager applied the $\mathcal{P} y \mathcal{F} \mathcal{S}$ in handling the collaborative-based recommender system [24]. In [25], Peng defines several distance, similarity, entropy and inclusion measures for $\mathcal{P} y \mathcal{F} \mathcal{S}$ and their relations between them.

Ashraf [26-28] defines the spherical fuzzy sets $\mathcal{S} \mathcal{F} \mathcal{S}$ s, which is the successful extension of picture fuzzy sets and $\mathcal{P} y \mathcal{F} \mathcal{S}$, by putting a new condition on positive membership $\xi$, neutral membership $\eta$ and negative membership functions $v$, i.e., $0 \leq \xi^{2}+\eta^{2}+v^{2} \leq 1$. This new condition expand the domain of membership functions like if we have $\xi=0.7, \eta=0.5$ and $v=0.5$, then we cannot deal it with picture fuzzy set because $0.7+0.5+0.5 \geq 1$ but $0.7^{2}+0.5^{2}+0.5^{2}=0.49+0.25+0.25=0.99 \leq 1$ and hence $\mathcal{S} \mathcal{F} \mathcal{S}$ applied successfully. In [29], Rafiq proposed similarity measure based on cosine and cotangent functions for $\mathcal{S} \mathcal{F} \mathcal{S} s$ and applied them to the pattern recognition. In [30], multi-attribute group decision making problem is solved by symmetric sum based aggregation operators for spherical fuzzy sets.

Keeping in mind the importance of similarity measure and application in data mining, medical diagnosis, decision making and pattern recognition many authors work on this topic. A wide theory of similarity measures of fuzzy sets and intuitionistic fuzzy sets are presented in the literature [31-34].

The generalization of $\mathcal{S} \mathcal{F} S \mathcal{S}$ is specified on $\mathcal{P F S}$ in a sense that the domains of membership, neutral and non-membership functions are grater in $\mathcal{S} \mathcal{F} \mathcal{S}_{S}$ i.e., the experts give their judgments more freely. In $\mathcal{S} \mathcal{F} \mathcal{S}$, the preferences of the experts describe precisely because it contains all aspects of assessment of information like yes, abstain, no and refusal. The generalization of $\mathcal{S} \mathcal{F} S s$ is specified on $\mathcal{P} y \mathcal{F} \mathcal{S}$ because it contains an extra degree of preferences: the neutral degree or neutral membership function.

The aim of this paper is to define the new similarity measures for $\mathcal{S F} \mathcal{S} S$ and discuss the selection of mega projects for under developing countries. Since, it is important for under developing countries to select upcoming mega projects on priority which has less effect on their economy, environment, less maintenance cost has long term benefits, fewer peoples effects from that project and generate high revenue. Normally, the megaprojects are characterized by vast complexity (especially in organizational terms), large investment commitment, long-lasting impact on the economy, the environment, and society. 
Some of the proposed similarity measures for $\mathcal{S} \mathcal{F} \mathcal{S}_{S}$ have some problems which are pointed out in Section 4. To improve the idea of the similarity measure, we proposed the set-theoretic similarity and distance measures. The proposed similarity measure is then applied to the pattern recognition. The selection of mega projects for under developing countries is done by the proposed similarity measure.

The remaining paper is organized as follows: Introduction and preliminaries are presented in Sections 1 and 2. In Section 3, we proposed the set-theoretic similarity measures for $\mathcal{S} \mathcal{F} \mathcal{S}$ s. In Section 4 , we provide some counterexamples for already proposed similarity measures. To support the proposed similarity measure a numerical example of selecting mega projects in under developing countries is presented in Section 5. Comparison analysis and conclusion are presented in Sections 6 and 7.

\section{Preliminaries}

In this section, we provide some basic definitions of $\mathcal{F} \mathcal{S}, \mathcal{I} \mathcal{F} \mathcal{S}, \mathcal{P} \mathcal{F} \mathcal{S}$, and $\mathcal{S} \mathcal{F}$. The already proposed similarity measures for $\mathcal{S} \mathcal{F} \mathcal{S}$ are discussed.

A fuzzy set is defined by Zadeh [1], which handles uncertainty based on the view of gradualness effectively.

Definition 1. [1] A membership function $\xi_{\hat{\mathcal{A}}}: \hat{\mathcal{Y}} \rightarrow[0,1]$ defines the fuzzy set $\hat{\mathcal{A}}$ over the $\hat{\mathcal{Y}}$, where $\xi_{\hat{\mathcal{A}}}(y)$ particularized the membership of an element $y \in \hat{\mathcal{Y}}$ in fuzzy set $\hat{\mathcal{A}}$.

In [10], Cuong defines the $\mathcal{P} \mathcal{F} \mathcal{S}$, which is an extension of a fuzzy set and applicable in many real-life problems. The picture fuzzy set is obtained by adding an extra membership function, namely, the degree of the neutral membership in $\mathcal{I F S}$. The information regarding the situation of type: yes, abstain, no and refusal can be model by using picture fuzzy set easily. Voting can be a good example of a picture fuzzy set because it involves the situation of more answers of the type: yes, abstain, no, refusal.

Definition 2. [10] $A \mathcal{P} \mathcal{F} \mathcal{S} \hat{\mathcal{A}}$ over the universe $\hat{\mathcal{Y}}$ is defined as

$$
\hat{\mathcal{A}}=\left\{\left(y, \xi_{\hat{\mathcal{A}}}, \eta_{\hat{\mathcal{A}}}, v_{\hat{\mathcal{A}}}\right) \mid y \in \hat{\mathcal{Y}}\right\},
$$

where $\xi_{\hat{\mathcal{A}}}: \hat{\mathcal{Y}} \rightarrow[0,1], \eta_{\hat{\mathcal{A}}}: \hat{\mathcal{Y}} \rightarrow[0,1]$ and $\vartheta_{\hat{\mathcal{A}}}: \hat{\mathcal{Y}} \rightarrow[0,1]$ are the degree of positive membership, neutral membership and degree of negative membership, respectively, such that $0 \leq \xi_{\hat{\mathcal{A}}}(y)+\eta_{\hat{\mathcal{A}}}(y)+v_{\hat{\mathcal{A}}}(y) \leq 1$.

Definition 3. [26] A SFS $\hat{\mathcal{A}}$ over the universe $\hat{\mathcal{Y}}$ is defined as

$$
\hat{\mathcal{A}}=\left\{\left(y, \xi_{\hat{\mathcal{A}}}, \eta_{\hat{\mathcal{A}}}, v_{\hat{\mathcal{A}}}\right) \mid y \in \hat{\mathcal{Y}}\right\},
$$

where $\xi_{\hat{\mathcal{A}}}: \hat{\mathcal{Y}} \rightarrow[0,1], \eta_{\hat{\mathcal{A}}}: \hat{\mathcal{Y}} \rightarrow[0,1]$ and $\vartheta_{\hat{\mathcal{A}}}: \hat{\mathcal{Y}} \rightarrow[0,1]$ are the degree of positive membership, neutral membership and degree of negative membership, respectively. Furthermore, it is required that $0 \leq \xi_{\hat{\mathcal{A}}}^{2}(y)+\eta_{\hat{\mathcal{A}}}^{2}(y)+v_{\hat{\mathcal{A}}}^{2}(y) \leq 1$. Then for $y \in \hat{\mathcal{Y}}, \pi_{\hat{\mathcal{A}}}(y)=\sqrt{1-\left(\xi_{\hat{\mathcal{A}}}^{2}(y)+\eta_{\hat{\mathcal{A}}}^{2}(y)+v_{\hat{\mathcal{A}}}^{2}(y)\right)}$ is called the degree of refusal membership of $y$ in $\hat{\mathcal{A}}$. For $\mathcal{S} \mathcal{F} \mathcal{S}\left(\xi_{\hat{\mathcal{A}}}(y), \eta_{\hat{\mathcal{A}}}(y), v_{\hat{\mathcal{A}}}(y)\right)$ are said to spherical fuzzy value $(S F V)$ or spherical fuzzy number $(S F N)$ and each $S F V$ can be denoted by $q=\left(\xi_{q}, \eta_{q}, v_{q}\right)$, where $\xi_{q}$, $\eta_{q}$ and $v_{q} \in[0,1]$, with condition that $0 \leq \xi_{q}^{2}+\eta_{q}^{2}+v_{q}^{2} \leq 1$. Therefore, the information regarding the situation of type: yes, abstain, no and refusal can be model more easily by using $\mathcal{S} \mathcal{F} \mathcal{S}$ than $\mathcal{P} \mathcal{F} \mathcal{S}$.

We can easily observed that the $\mathcal{S} \mathcal{F} \mathcal{S}$ is an extension of $\mathcal{P} \mathcal{F} \mathcal{S}$. For example, if we have $\xi=0.6, \eta=0.5$ and $v=0.4$, then $0.6+0.5+0.4=1.5>1$. However, $0.6^{2}+0.5^{2}+0.4^{2}=0.36+0.25+0.16=0.77<1$, hence $\mathcal{S} \mathcal{F} \mathcal{S}$ expand the domain of memberships functions.

In [29], Rafiq defines some similarity measures for $\mathcal{S} \mathcal{F} \mathcal{S}$ s based on cosine and cotangent functions. 
Definition 4. [29] For two $\mathcal{S} \mathcal{F} \mathcal{S} S \hat{\mathcal{A}}$ and $\hat{\mathcal{B}}$ in $\hat{\mathcal{Y}}$, a cosine similarity measure between $\hat{\mathcal{A}}$ and $\hat{\mathcal{B}}$ is defined as follows:

$$
\mathcal{S}_{c}^{1}(\hat{\mathcal{A}}, \hat{\mathcal{B}})=\frac{1}{m} \sum_{j=1}^{m} \frac{\xi_{\hat{\mathcal{A}}}^{2}\left(y_{j}\right) \xi_{\hat{\mathcal{B}}}^{2}\left(y_{j}\right)+\eta_{\hat{\mathcal{A}}}^{2}\left(y_{j}\right) \eta_{\hat{\mathcal{B}}}^{2}\left(y_{j}\right)+v_{\hat{\mathcal{A}}}^{2}\left(y_{j}\right) v_{\hat{\mathcal{B}}}^{2}\left(y_{j}\right)}{\sqrt{\xi_{\hat{\mathcal{A}}}^{4}\left(y_{j}\right)+\eta_{\hat{\mathcal{A}}}^{4}\left(y_{j}\right)+v_{\hat{\mathcal{A}}}^{4}\left(y_{j}\right)} \sqrt{\xi_{\hat{\mathcal{B}}}^{4}\left(y_{j}\right)+\eta_{\hat{\mathcal{B}}}^{4}\left(y_{j}\right)+v_{\hat{\mathcal{B}}}^{4}\left(y_{j}\right)}} .
$$

Definition 5. [29] For two $\mathcal{S} \mathcal{F} \mathcal{S} S \hat{\mathcal{A}}$ and $\hat{\mathcal{B}}$ in $\hat{\mathcal{Y}}$, similarity measures using cosine function between $\hat{\mathcal{A}}$ and $\hat{\mathcal{B}}$ are defined as follows:

$$
\begin{aligned}
& \mathcal{S}_{c}^{2}(\hat{\mathcal{A}}, \hat{\mathcal{B}})=\frac{1}{m} \sum_{j=1}^{m} \cos \left\{\frac{\pi}{2}\left[\left|\xi_{\hat{\mathcal{A}}}^{2}\left(y_{j}\right)-\xi_{\hat{\mathcal{B}}}^{2}\left(y_{j}\right)\right| \vee\left|\eta_{\hat{\mathcal{A}}}^{2}\left(y_{j}\right)-\eta_{\hat{\mathcal{B}}}^{2}\left(y_{j}\right)\right| \vee\left|v_{\hat{\mathcal{A}}}^{2}\left(y_{j}\right)-v_{\hat{\mathcal{B}}}^{2}\left(y_{j}\right)\right|\right]\right\} \\
& \mathcal{S}_{c}^{3}(\hat{\mathcal{A}}, \hat{\mathcal{B}})=\frac{1}{m} \sum_{j=1}^{m} \cos \left\{\frac{\pi}{4}\left[\left|\xi_{\hat{\mathcal{A}}}^{2}\left(y_{j}\right)-\xi_{\hat{\mathcal{B}}}^{2}\left(y_{j}\right)\right|+\left|\eta_{\hat{\mathcal{A}}}^{2}\left(y_{j}\right)-\eta_{\hat{\mathcal{B}}}^{2}\left(y_{j}\right)\right|+\left|v_{\hat{\mathcal{A}}}^{2}\left(y_{j}\right)-v_{\hat{\mathcal{B}}}^{2}\left(y_{j}\right)\right|\right]\right\}
\end{aligned}
$$

where $\vee$ is the maximum operation.

Definition 6. [29] For two $\mathcal{S} \mathcal{F} \mathcal{S} S \hat{\mathcal{A}}$ and $\hat{\mathcal{B}}$ in $\hat{\mathcal{Y}}$, a cotangent similarity measure between $\hat{\mathcal{A}}$ and $\hat{\mathcal{B}}$ are defined as follows:

$$
\begin{aligned}
& \mathcal{S}_{c}^{4}(\hat{\mathcal{A}}, \hat{\mathcal{B}})=\frac{1}{m} \sum_{j=1}^{m} \cot \left\{\frac{\pi}{4}+\frac{\pi}{4}\left[\left|\xi_{\hat{\mathcal{A}}}^{2}\left(y_{j}\right)-\xi_{\hat{\mathcal{B}}}^{2}\left(y_{j}\right)\right| \vee\left|\eta_{\hat{\mathcal{A}}}^{2}\left(y_{j}\right)-\eta_{\hat{\mathcal{B}}}^{2}\left(y_{j}\right)\right| \vee\left|v_{\hat{\mathcal{A}}}^{2}\left(y_{j}\right)-v_{\hat{\mathcal{B}}}^{2}\left(y_{j}\right)\right|\right]\right\} \\
& \mathcal{S}_{c}^{5}(\hat{\mathcal{A}}, \hat{\mathcal{B}})=\frac{1}{m} \sum_{j=1}^{m} \cot \left\{\frac{\pi}{4}+\frac{\pi}{8}\left[\left|\xi_{\hat{\mathcal{A}}}^{2}\left(y_{j}\right)-\xi_{\hat{\mathcal{B}}}^{2}\left(y_{j}\right)\right|+\left|\eta_{\hat{\mathcal{A}}}^{2}\left(y_{j}\right)-\eta_{\mathcal{B}}^{2}\left(y_{j}\right)\right|+\left|v_{\hat{\mathcal{A}}}^{2}\left(y_{j}\right)-v_{\hat{\mathcal{B}}}^{2}\left(y_{j}\right)\right|\right]\right\}
\end{aligned}
$$

where $\vee$ is the maximum operation.

Definition 7. [29] For two $\mathcal{S} \mathcal{F} S$ s $\hat{\mathcal{A}}$ and $\hat{\mathcal{B}}$ in $\hat{\mathcal{Y}}$, a cosine similarity measure by using degree of refusal membership between $\hat{\mathcal{A}}$ and $\hat{\mathcal{B}}$ are defined as follows:

$$
\begin{aligned}
& \mathcal{S}_{c}^{6}(\hat{\mathcal{A}}, \hat{\mathcal{B}})= \\
& \frac{1}{m} \sum_{j=1}^{m} \cos \left\{\frac{\pi}{2}\left[\left|\xi_{\hat{\mathcal{A}}}^{2}\left(y_{j}\right)-\xi_{\hat{\mathcal{B}}}^{2}\left(y_{j}\right)\right| \vee\left|\eta_{\hat{\mathcal{A}}}^{2}\left(y_{j}\right)-\eta_{\hat{\mathcal{B}}}^{2}\left(y_{j}\right)\right| \vee\left|v_{\hat{\mathcal{A}}}^{2}\left(y_{j}\right)-v_{\hat{\mathcal{B}}}^{2}\left(y_{j}\right)\right| \vee\left|\pi_{\hat{\mathcal{A}}}\left(y_{j}\right)-\pi_{\mathcal{B}}\left(y_{j}\right)\right|\right]\right\} \\
& \mathcal{S}_{c}^{7}(\hat{\mathcal{A}}, \hat{\mathcal{B}})= \\
& \frac{1}{m} \sum_{j=1}^{m} \cos \left\{\frac{\pi}{4}\left[\left|\xi_{\hat{\mathcal{A}}}^{2}\left(y_{j}\right)-\xi_{\hat{\mathcal{B}}}^{2}\left(y_{j}\right)\right|+\left|\eta_{\hat{\mathcal{A}}}^{2}\left(y_{j}\right)-\eta_{\hat{\mathcal{B}}}^{2}\left(y_{j}\right)\right|+\left|v_{\hat{\mathcal{A}}}^{2}\left(y_{j}\right)-v_{\hat{\mathcal{B}}}^{2}\left(y_{j}\right)\right|+\left|\pi_{\hat{\mathcal{A}}}\left(y_{j}\right)-\pi_{\hat{\mathcal{B}}}\left(y_{j}\right)\right|\right]\right\}
\end{aligned}
$$

where $\vee$ is the maximum operation.

Definition 8. [29] For two $\mathcal{S} \mathcal{F} \mathcal{S} s \hat{\mathcal{A}}$ and $\hat{\mathcal{B}}$ in $\hat{\mathcal{Y}}$, a cotangent similarity measure by using degree of refusal membership between $\hat{\mathcal{A}}$ and $\hat{\mathcal{B}}$ is defined as follows:

$$
\begin{aligned}
& \mathcal{S}_{c}^{8}(\hat{\mathcal{A}}, \hat{\mathcal{B}})= \\
& \frac{1}{m} \sum_{j=1}^{m} \cot \left\{\frac{\pi}{4}+\frac{\pi}{4}\left[\left|\xi_{\hat{\mathcal{A}}}^{2}\left(y_{j}\right)-\xi_{\mathcal{B}}^{2}\left(y_{j}\right)\right| \vee\left|\eta_{\hat{\mathcal{A}}}^{2}\left(y_{j}\right)-\eta_{\hat{\mathcal{B}}}^{2}\left(y_{j}\right)\right| \vee\left|v_{\hat{\mathcal{A}}}^{2}\left(y_{j}\right)-v_{\mathcal{B}}^{2}\left(y_{j}\right)\right| \vee\left|\pi_{\hat{\mathcal{A}}}\left(y_{j}\right)-\pi_{\hat{\mathcal{B}}}\left(y_{j}\right)\right|\right]\right\} \\
& \mathcal{S}_{c}^{9}(\hat{\mathcal{A}}, \hat{\mathcal{B}})= \\
& \frac{1}{m} \sum_{j=1}^{m} \cot \left\{\frac{\pi}{4}+\frac{\pi}{8}\left[\left|\xi_{\hat{\mathcal{A}}}^{2}\left(y_{j}\right)-\tilde{\xi}_{\hat{\mathcal{B}}}^{2}\left(y_{j}\right)\right|+\left|\eta_{\hat{\mathcal{A}}}^{2}\left(y_{j}\right)-\eta_{\hat{\mathcal{B}}}^{2}\left(y_{j}\right)\right|+\left|v_{\hat{\mathcal{A}}}^{2}\left(y_{j}\right)-v_{\hat{\mathcal{B}}}^{2}\left(y_{j}\right)\right|+\left|\pi_{\hat{\mathcal{A}}}\left(y_{j}\right)-\pi_{\hat{\mathcal{B}}}\left(y_{j}\right)\right|\right]\right\}
\end{aligned}
$$

where $\vee$ is the maximum operation. 


\section{A New Similarity Measures for $\mathcal{S} \mathcal{F} \mathcal{S}_{S}$}

In this section, we define new similarity and distance measures for $\mathcal{S} \mathcal{F} \mathcal{S}$ s give their proof.

Definition 9. A distance measure between $\mathcal{S} \mathcal{F} \mathcal{S} S \hat{\mathcal{A}}$ and $\hat{\mathcal{B}}$ is a mapping $\hat{\mathcal{D}}: S F S \times S F S \rightarrow[0,1]$, which satisfies the following properties:

(D1) $0 \leq \hat{\mathcal{D}}(\hat{\mathcal{A}}, \hat{\mathcal{B}}) \leq 1$

(D2) $\hat{\mathcal{D}}(\hat{\mathcal{A}}, \hat{\mathcal{B}})=0 \Longleftrightarrow \hat{\mathcal{A}}=\hat{\mathcal{B}}$

(D3) $\hat{\mathcal{D}}(\hat{\mathcal{A}}, \hat{\mathcal{B}})=\hat{\mathcal{D}}(\hat{\mathcal{B}}, \hat{\mathcal{A}})$

(D4) If $\hat{\mathcal{A}} \subseteq \hat{\mathcal{B}} \subseteq \hat{\mathcal{C}}$ then $\hat{\mathcal{D}}(\hat{\mathcal{A}}, \hat{\mathcal{C}}) \geq \hat{\mathcal{D}}(\hat{\mathcal{A}}, \hat{\mathcal{B}})$ and $\hat{\mathcal{D}}(\hat{\mathcal{A}}, \hat{\mathcal{C}}) \geq \hat{\mathcal{D}}(\hat{\mathcal{B}}, \hat{\mathcal{C}})$.

Definition 10. A similarity measure between $\mathcal{S} \mathcal{F} \mathcal{S} S \hat{\mathcal{A}}$ and $\hat{\mathcal{B}}$ is a mapping $\hat{\mathcal{S}}: S F S \times S F S \rightarrow[0,1]$, which satisfies the following properties:

(S1) $0 \leq \hat{\mathcal{S}}(\hat{\mathcal{A}}, \hat{\mathcal{B}}) \leq 1$

(S2) $\hat{\mathcal{S}}(\hat{\mathcal{A}}, \hat{\mathcal{B}})=1 \Longleftrightarrow \hat{\mathcal{A}}=\hat{\mathcal{B}}$

(S3) $\hat{\mathcal{S}}(\hat{\mathcal{A}}, \hat{\mathcal{B}})=\hat{\mathcal{S}}(\hat{\mathcal{B}}, \hat{\mathcal{A}})$

(S4) If $\hat{\mathcal{A}} \subseteq \hat{\mathcal{B}} \subseteq \hat{\mathcal{C}}$ then $\hat{\mathcal{S}}(\hat{\mathcal{A}}, \hat{\mathcal{C}}) \leq \hat{\mathcal{S}}(\hat{\mathcal{A}}, \hat{\mathcal{B}})$ and $\hat{\mathcal{S}}(\hat{\mathcal{A}}, \hat{\mathcal{C}}) \leq \hat{\mathcal{S}}(\hat{\mathcal{B}}, \hat{\mathcal{C}})$.

Definition 11. For two $\mathcal{S} \mathcal{F} \mathcal{S} S \hat{\mathcal{A}}$ and $\hat{\mathcal{B}}$ in $\hat{\mathcal{Y}}$, a new similarity measures is defined between $\hat{\mathcal{A}}$ and $\hat{\mathcal{B}}$ as follows:

$$
\mathcal{S}^{s}(\hat{\mathcal{A}}, \hat{\mathcal{B}})=\frac{\sum_{j=1}^{m}\left[\xi_{\hat{\mathcal{A}}}^{2}\left(y_{j}\right) \cdot \xi_{\hat{\mathcal{B}}}^{2}\left(y_{j}\right)+\eta_{\hat{\mathcal{A}}}^{2}\left(y_{j}\right) \cdot \eta_{\hat{\mathcal{B}}}^{2}\left(y_{j}\right)+v_{\hat{\mathcal{A}}}^{2}\left(y_{j}\right) \cdot v_{\hat{\mathcal{B}}}^{2}\left(y_{j}\right)\right]}{\sum_{j=1}^{m}\left[\left\{\xi_{\hat{\mathcal{A}}}^{4}\left(y_{j}\right) \vee \xi_{\hat{\mathcal{B}}}^{4}\left(y_{j}\right)\right\}+\left\{\eta_{\hat{\mathcal{A}}}^{4}\left(y_{j}\right) \vee \eta_{\hat{\mathcal{B}}}^{4}\left(y_{j}\right)\right\}+\left\{v_{\hat{\mathcal{A}}}^{4}\left(y_{j}\right) \vee v_{\hat{\mathcal{B}}}^{4}\left(y_{j}\right)\right\}\right]} .
$$

Example 1. Let $\hat{\mathcal{Y}}=\left\{y_{1}, y_{2}, y_{3}, y_{4}, y_{5}\right\}$ be the universal set. We consider two $\mathcal{S} \mathcal{F} \mathcal{S}$ s $\hat{\mathcal{A}}$ and $\hat{\mathcal{B}}$ in $\hat{\mathcal{Y}}$, which are given as follows:

$$
\begin{gathered}
\hat{\mathcal{A}}=\left\{(0.7,0.1,0.2) / y_{1},(0.7,0.2,0.2) / y_{2},(0.2,0.1,0.7) / y_{3},(0.9,0.1,0.2) / y_{4},(0.2,0.1,0.6) / y_{5}\right\} \\
\hat{\mathcal{B}}=\left\{(0.3,0.2,0.4) / y_{1},(0.5,0.2,0.1) / y_{2},(0.1,0.1,0.7) / y_{3},(0.4,0.1,0.3) / y_{4},(0.1,0.1,0.7) / y_{5}\right\} . \\
\mathcal{S}^{S}(\hat{\mathcal{A}}, \hat{\mathcal{B}})=\frac{0.0509+0.1245+0.2406+0.1333+0.1769}{0.2673+0.2433+0.2418+0.6643+0.2418}=\frac{0.61}{0.91}=0.669594
\end{gathered}
$$

Theorem 1. $\mathcal{S}^{S}(\hat{\mathcal{A}}, \hat{\mathcal{B}})$ is the similarity measure between two $\mathcal{S F} \mathcal{S}$ s $\hat{\mathcal{A}}$ and $\hat{\mathcal{B}}$ in $\hat{\mathcal{Y}}$.

Proof. To prove $\mathcal{S}^{s}$ a similarity measure, we have to verify the four conditions of Definition 10 for $\mathcal{S}^{s}$.

(S1). Since for all $y_{j}, 1 \leq j \leq m$, we have $\xi_{\hat{\mathcal{A}}}^{2}\left(y_{j}\right) \cdot \xi_{\hat{\mathcal{B}}}^{2}\left(y_{j}\right) \leq \xi_{\hat{\mathcal{A}}}^{4}\left(y_{j}\right) \vee \xi_{\hat{\mathcal{B}}}^{4}\left(y_{j}\right), \eta_{\hat{\mathcal{A}}}^{2}\left(y_{j}\right) \cdot \eta_{\hat{\mathcal{B}}}^{2}\left(y_{j}\right) \leq$ $\eta_{\hat{\mathcal{A}}}^{4}\left(y_{j}\right) \vee \eta_{\hat{\mathcal{B}}}^{4}\left(y_{j}\right)$ and $v_{\hat{\mathcal{A}}}^{2}\left(y_{j}\right) \cdot v_{\hat{\mathcal{B}}}^{2}\left(y_{j}\right) \leq v_{\hat{\mathcal{A}}}^{4}\left(y_{j}\right) \vee v_{\hat{\mathcal{B}}}^{4}\left(y_{j}\right)$. Therefore for each $y_{j}$, we have

$$
\begin{aligned}
{\left[\xi_{\hat{\mathcal{A}}}^{2}\left(y_{j}\right) \cdot \xi_{\hat{\mathcal{B}}}^{2}\left(y_{j}\right)+\eta_{\hat{\mathcal{A}}}^{2}\left(y_{j}\right) \cdot \eta_{\hat{\mathcal{B}}}^{2}\left(y_{j}\right)+\right.} & \left.v_{\hat{\mathcal{A}}}^{2}\left(y_{j}\right) \cdot v_{\hat{\mathcal{B}}}^{2}\left(y_{j}\right)\right] \\
& \leq\left[\left\{\xi_{\hat{\mathcal{A}}}^{4}\left(y_{j}\right) \vee \xi_{\hat{\mathcal{B}}}^{4}\left(y_{j}\right)\right\}+\left\{\eta_{\hat{\mathcal{A}}}^{4}\left(y_{j}\right) \vee \eta_{\hat{\mathcal{B}}}^{4}\left(y_{j}\right)\right\}+\left\{v_{\hat{\mathcal{A}}}^{4}\left(y_{j}\right) \vee v_{\hat{\mathcal{B}}}^{4}\left(y_{j}\right)\right\}\right] .
\end{aligned}
$$

Therefore for all $y_{j}, 1 \leq j \leq m$, we have

$$
\begin{aligned}
\sum_{j=1}^{m}\left[\xi_{\hat{\mathcal{A}}}^{2}\left(y_{j}\right) \cdot \xi_{\hat{\mathcal{B}}}^{2}\left(y_{j}\right)+\eta_{\hat{\mathcal{A}}}^{2}\left(y_{j}\right)\right. & \left.\cdot \eta_{\hat{\mathcal{B}}}^{2}\left(y_{j}\right)+v_{\hat{\mathcal{A}}}^{2}\left(y_{j}\right) \cdot v_{\hat{\mathcal{B}}}^{2}\left(y_{j}\right)\right] \\
& \leq \sum_{j=1}^{m}\left[\left\{\xi_{\hat{\mathcal{A}}}^{4}\left(y_{j}\right) \vee \xi_{\hat{\mathcal{B}}}^{4}\left(y_{j}\right)\right\}+\left\{\eta_{\hat{\mathcal{A}}}^{4}\left(y_{j}\right) \vee \eta_{\hat{\mathcal{B}}}^{4}\left(y_{j}\right)\right\}+\left\{v_{\hat{\mathcal{A}}}^{4}\left(y_{j}\right) \vee v_{\hat{\mathcal{B}}}^{4}\left(y_{j}\right)\right\}\right] \\
0 & \leq \mathcal{S}^{\mathcal{S}}(\hat{\mathcal{A}}, \hat{\mathcal{B}}) \leq 1
\end{aligned}
$$


(S2). Suppose $\mathcal{S}^{s}(\hat{\mathcal{A}}, \hat{\mathcal{B}})=1$. We have to prove $\hat{\mathcal{A}}=\hat{\mathcal{B}}$. By definition of $\mathcal{S}^{s}$,

$$
\begin{gathered}
\frac{\sum_{j=1}^{m}\left[\xi_{\hat{\mathcal{A}}}^{2}\left(y_{j}\right) \cdot \xi_{\hat{\mathcal{B}}}^{2}\left(y_{j}\right)+\eta_{\hat{\mathcal{A}}}^{2}\left(y_{j}\right) \cdot \eta_{\hat{\mathcal{B}}}^{2}\left(y_{j}\right)+v_{\hat{\mathcal{A}}}^{2}\left(y_{j}\right) \cdot v_{\hat{\mathcal{B}}}^{2}\left(y_{j}\right)\right]}{\sum_{j=1}^{m}\left[\left\{\xi_{\hat{\mathcal{A}}}^{4}\left(y_{j}\right) \vee \xi_{\hat{\mathcal{B}}}^{4}\left(y_{j}\right)\right\}+\left\{\eta_{\hat{\mathcal{A}}}^{4}\left(y_{j}\right) \vee \eta_{\mathcal{B}}^{4}\left(y_{j}\right)\right\}+\left\{v_{\hat{\mathcal{A}}}^{4}\left(y_{j}\right) \vee v_{\mathcal{B}}^{4}\left(y_{j}\right)\right\}\right]}=1 \\
\Rightarrow \sum_{j=1}^{m}\left[\xi_{\hat{\mathcal{A}}}^{2}\left(y_{j}\right) \cdot \xi_{\hat{\mathcal{B}}}^{2}\left(y_{j}\right)+\eta_{\hat{\mathcal{A}}}^{2}\left(y_{j}\right) \cdot \eta_{\hat{\mathcal{B}}}^{2}\left(y_{j}\right)+v_{\hat{\mathcal{A}}}^{2}\left(y_{j}\right) \cdot v_{\mathcal{B}}^{2}\left(y_{j}\right)\right] \\
=\sum_{j=1}^{m}\left[\left\{\xi_{\hat{\mathcal{A}}}^{4}\left(y_{j}\right) \vee \xi_{\mathcal{B}}^{4}\left(y_{j}\right)\right\}+\left\{\eta_{\hat{\mathcal{A}}}^{4}\left(y_{j}\right) \vee \eta_{\mathcal{B}}^{4}\left(y_{j}\right)\right\}+\left\{v_{\hat{\mathcal{A}}}^{4}\left(y_{j}\right) \vee v_{\hat{\mathcal{B}}}^{4}\left(y_{j}\right)\right\}\right] .
\end{gathered}
$$

Now we claim that $\xi_{\hat{\mathcal{A}}}^{2}\left(y_{j}\right) \cdot \xi_{\hat{\mathcal{B}}}^{2}\left(y_{j}\right)=\xi_{\hat{\mathcal{A}}}^{4}\left(y_{j}\right) \vee \xi_{\hat{\mathcal{B}}}^{4}\left(y_{j}\right), \eta_{\hat{\mathcal{A}}}^{2}\left(y_{j}\right) \cdot \eta_{\hat{\mathcal{B}}}^{2}\left(y_{j}\right)=\eta_{\hat{\mathcal{A}}}^{4}\left(y_{j}\right) \vee \eta_{\hat{\mathcal{B}}}^{4}\left(y_{j}\right)$ and $v_{\hat{\mathcal{A}}}^{2}\left(y_{j}\right)$. $v_{\hat{\mathcal{B}}}^{2}\left(y_{j}\right)=v_{\hat{\mathcal{A}}}^{4}\left(y_{j}\right) \vee v_{\mathcal{B}}^{4}\left(y_{j}\right)$.

Suppose $\xi_{\hat{\mathcal{A}}}^{2}\left(y_{j}\right) \cdot \xi_{\hat{\mathcal{B}}}^{2}\left(y_{j}\right) \neq \xi_{\hat{\mathcal{A}}}^{4}\left(y_{j}\right) \vee \xi_{\hat{\mathcal{B}}}^{4}\left(y_{j}\right)$, since $\xi_{\hat{\mathcal{A}}}^{2}\left(y_{j}\right) \cdot \xi_{\hat{\mathcal{B}}}^{2}\left(y_{j}\right) \leq \xi_{\hat{\mathcal{A}}}^{4}\left(y_{j}\right) \vee \xi_{\hat{\mathcal{B}}}^{4}\left(y_{j}\right)$, there exists $r>0$ such that $\tilde{\zeta}_{\hat{\mathcal{A}}}^{2}\left(y_{j}\right) \cdot \xi_{\hat{\mathcal{B}}}^{2}\left(y_{j}\right)+r=\xi_{\hat{\mathcal{A}}}^{4}\left(y_{j}\right) \vee \xi_{\hat{\mathcal{B}}}^{4}\left(y_{j}\right)$.

Similarly there exists $s, t>0$ such that $\eta_{\hat{\mathcal{A}}}^{2}\left(y_{j}\right) \cdot \eta_{\hat{\mathcal{B}}}^{2}\left(y_{j}\right)+s=\eta_{\hat{\mathcal{A}}}^{4}\left(y_{j}\right) \vee \eta_{\mathcal{B}}^{4}\left(y_{j}\right)$ and $v_{\hat{\mathcal{A}}}^{2}\left(y_{j}\right) \cdot v_{\hat{\mathcal{B}}}^{2}\left(y_{j}\right)+$ $t=v_{\hat{\mathcal{A}}}^{4}\left(y_{j}\right) \vee v_{\mathcal{B}}^{4}\left(y_{j}\right)$.

By hypothesis it follows that $r+s+t=0$. This implies that $r=-(s+t)$, which is not possible. This implies that $\xi_{\hat{\mathcal{A}}}^{2}\left(y_{j}\right) \cdot \xi_{\hat{\mathcal{B}}}^{2}\left(y_{j}\right)=\xi_{\hat{\mathcal{A}}}^{4}\left(y_{j}\right) \vee \xi_{\hat{\mathcal{B}}}^{4}\left(y_{j}\right), \eta_{\hat{\mathcal{A}}}^{2}\left(y_{j}\right) \cdot \eta_{\hat{\mathcal{B}}}^{2}\left(y_{j}\right)=\eta_{\hat{\mathcal{A}}}^{4}\left(y_{j}\right) \vee \eta_{\hat{\mathcal{B}}}^{4}\left(y_{j}\right)$ and $v_{\hat{\mathcal{A}}}^{2}\left(y_{j}\right)$. $v_{\hat{\mathcal{B}}}^{2}\left(y_{j}\right)=v_{\hat{\mathcal{A}}}^{4}\left(y_{j}\right) \vee v_{\hat{\mathcal{B}}}^{4}\left(y_{j}\right)$. This implies that $\xi_{\hat{\mathcal{A}}}^{2}\left(y_{j}\right)=\tilde{\xi}_{\hat{\mathcal{B}}}^{2}\left(y_{j}\right), \eta_{\hat{\mathcal{A}}}^{2}\left(y_{j}\right)=\eta_{\mathcal{B}}^{2}\left(y_{j}\right)$ and $v_{\hat{\mathcal{A}}}^{2}\left(y_{j}\right)=v_{\hat{\mathcal{B}}}^{2}\left(y_{j}\right)$. Hence $\hat{\mathcal{A}}=\hat{\mathcal{B}}$.

Converse, trivially follows from Definition 11.

(S3). $\mathcal{S}^{s}(\hat{\mathcal{A}}, \hat{\mathcal{B}})=\mathcal{S}^{s}(\hat{\mathcal{B}}, \hat{\mathcal{A}})$ is trivial.

(S4). For three $\mathcal{S} \mathcal{F} \mathcal{S} S \hat{\mathcal{A}}, \hat{\mathcal{B}}$ and $\hat{\mathcal{C}}$ in $\hat{\mathcal{Y}}$. The similarity measures between $\hat{\mathcal{A}}, \hat{\mathcal{B}}$ and $\hat{\mathcal{A}}, \hat{\mathcal{C}}$ are given as:

$$
\begin{aligned}
& \mathcal{S}^{\mathcal{S}}(\hat{\mathcal{A}}, \hat{\mathcal{B}})=\frac{\sum_{j=1}^{m}\left[\xi_{\hat{\mathcal{A}}}^{2}\left(y_{j}\right) \cdot \xi_{\hat{\mathcal{B}}}^{2}\left(y_{j}\right)+\eta_{\hat{\mathcal{A}}}^{2}\left(y_{j}\right) \cdot \eta_{\hat{\mathcal{B}}}^{2}\left(y_{j}\right)+v_{\hat{\mathcal{A}}}^{2}\left(y_{j}\right) \cdot v_{\hat{\mathcal{B}}}^{2}\left(y_{j}\right)\right]}{\sum_{j=1}^{m}\left[\left\{\xi_{\hat{\mathcal{A}}}^{4}\left(y_{j}\right) \vee \xi_{\hat{\mathcal{B}}}^{4}\left(y_{j}\right)\right\}+\left\{\eta_{\hat{\mathcal{A}}}^{4}\left(y_{j}\right) \vee \eta_{\hat{\mathcal{B}}}^{4}\left(y_{j}\right)\right\}+\left\{v_{\hat{\mathcal{A}}}^{4}\left(y_{j}\right) \vee v_{\hat{\mathcal{B}}}^{4}\left(y_{j}\right)\right\}\right]} . \\
& \mathcal{S}^{\mathcal{S}}(\hat{\mathcal{A}}, \hat{\mathcal{C}})=\frac{\sum_{j=1}^{m}\left[\xi_{\hat{\mathcal{A}}}^{2}\left(y_{j}\right) \cdot \xi_{\hat{\mathcal{C}}}^{2}\left(y_{j}\right)+\eta_{\hat{\mathcal{A}}}^{2}\left(y_{j}\right) \cdot \eta_{\hat{\mathcal{C}}}^{2}\left(y_{j}\right)+v_{\hat{\mathcal{A}}}^{2}\left(y_{j}\right) \cdot v_{\hat{\mathcal{C}}}^{2}\left(y_{j}\right)\right]}{\sum_{j=1}^{m}\left[\left\{\xi_{\hat{\mathcal{A}}}^{4}\left(y_{j}\right) \vee \xi_{\hat{\mathcal{C}}}^{4}\left(y_{j}\right)\right\}+\left\{\eta_{\hat{\mathcal{A}}}^{4}\left(y_{j}\right) \vee \eta_{\hat{\mathcal{C}}}^{4}\left(y_{j}\right)\right\}+\left\{v_{\hat{\mathcal{A}}}^{4}\left(y_{j}\right) \vee v_{\hat{\mathcal{C}}}^{4}\left(y_{j}\right)\right\}\right]} .
\end{aligned}
$$

Suppose $\hat{\mathcal{A}} \subseteq \hat{\mathcal{B}} \subseteq \hat{\mathcal{C}}$. For all $y_{j} \in \hat{\mathcal{Y}}$, we have $\xi_{\hat{\mathcal{A}}}^{2}\left(y_{j}\right) \leq \xi_{\hat{\mathcal{B}}}^{2}\left(y_{j}\right) \leq \xi_{\hat{\mathcal{C}}}^{2}\left(y_{j}\right), \eta_{\hat{\mathcal{A}}}^{2}\left(y_{j}\right) \leq \eta_{\hat{\mathcal{B}}}^{2}\left(y_{j}\right) \leq \eta_{\hat{\mathcal{C}}}^{2}\left(y_{j}\right)$ and $v_{\hat{\mathcal{A}}}^{2}\left(y_{j}\right) \geq v_{\hat{\mathcal{B}}}^{2}\left(y_{j}\right) \geq v_{\hat{\mathcal{C}}}^{2}\left(y_{j}\right)$. This implies that $\xi_{\hat{\mathcal{A}}}^{4}\left(y_{j}\right) \leq \xi_{\hat{\mathcal{B}}}^{4}\left(y_{j}\right) \leq \xi_{\hat{\mathcal{C}}}^{4}\left(y_{j}\right), \eta_{\hat{\mathcal{A}}}^{4}\left(y_{j}\right) \leq \eta_{\hat{\mathcal{B}}}^{4}\left(y_{j}\right) \leq \eta_{\hat{\mathcal{C}}}^{4}\left(y_{j}\right)$ and $v_{\hat{\mathcal{A}}}^{4}\left(y_{j}\right) \geq v_{\hat{\mathcal{B}}}^{4}\left(y_{j}\right) \geq v_{\hat{\mathcal{C}}}^{4}\left(y_{j}\right)$. Then we have

$$
\begin{aligned}
\mathcal{S}^{\mathcal{S}}(\hat{\mathcal{A}}, \hat{\mathcal{B}}) & =\frac{\sum_{j=1}^{m}\left[\xi_{\hat{\mathcal{A}}}^{2}\left(y_{j}\right) \cdot \xi_{\hat{\mathcal{B}}}^{2}\left(y_{j}\right)+\eta_{\hat{\mathcal{A}}}^{2}\left(y_{j}\right) \cdot \eta_{\hat{\mathcal{B}}}^{2}\left(y_{j}\right)+v_{\hat{\mathcal{A}}}^{2}\left(y_{j}\right) \cdot v_{\hat{\mathcal{B}}}^{2}\left(y_{j}\right)\right]}{\sum_{j=1}^{m}\left[\left\{\xi_{\hat{\mathcal{B}}}^{4}\left(y_{j}\right)\right\}+\left\{\eta_{\hat{\mathcal{B}}}^{4}\left(y_{j}\right)\right\}+\left\{v_{\hat{\mathcal{A}}}^{4}\left(y_{j}\right)\right\}\right]}, \\
\mathcal{S}^{\mathcal{S}}(\hat{\mathcal{A}}, \hat{\mathcal{C}}) & =\frac{\sum_{j=1}^{m}\left[\xi_{\hat{\mathcal{A}}}^{2}\left(y_{j}\right) \cdot \xi_{\hat{\mathcal{C}}}^{2}\left(y_{j}\right)+\eta_{\hat{\mathcal{A}}}^{2}\left(y_{j}\right) \cdot \eta_{\hat{\mathcal{C}}}^{2}\left(y_{j}\right)+v_{\hat{\mathcal{A}}}^{2}\left(y_{j}\right) \cdot v_{\hat{\mathcal{C}}}^{2}\left(y_{j}\right)\right]}{\sum_{j=1}^{m}\left[\left\{\xi_{\hat{\mathcal{C}}}^{4}\left(y_{j}\right)\right\}+\left\{\eta_{\hat{\mathcal{C}}}^{4}\left(y_{j}\right)\right\}+\left\{v_{\hat{\mathcal{A}}}^{4}\left(y_{j}\right)\right\}\right]} .
\end{aligned}
$$


We claim that for all $y_{j} \in \hat{\mathcal{Y}}$, we have

$$
\frac{\xi_{\hat{\mathcal{A}}}^{2}\left(y_{j}\right) \cdot \xi_{\hat{\mathcal{B}}}^{2}\left(y_{j}\right)}{\xi_{\mathcal{B}}^{4}\left(y_{j}\right)+\eta_{\mathcal{B}}^{4}\left(y_{j}\right)+v_{\hat{\mathcal{A}}}^{4}\left(y_{j}\right)} \geq \frac{\xi_{\hat{\mathcal{A}}}^{2}\left(y_{j}\right) \cdot \xi_{\hat{\mathcal{C}}}^{2}\left(y_{j}\right)}{\xi_{\mathcal{C}}^{4}\left(y_{j}\right)+\eta_{\mathcal{C}}^{4}\left(y_{j}\right)+v_{\hat{\mathcal{A}}}^{4}\left(y_{j}\right)},
$$

because $\eta_{\hat{\mathcal{B}}}^{4}\left(y_{j}\right) \leq \eta_{\hat{\mathcal{C}}}^{4}\left(y_{j}\right)$ and $\frac{1}{\xi_{\hat{\mathcal{B}}}^{2}\left(y_{j}\right)} \geq \frac{1}{\xi_{\hat{\mathcal{C}}}^{2}\left(y_{j}\right)}$. Similarly, we have

$$
\begin{aligned}
& \frac{\eta_{\hat{\mathcal{A}}}^{2}\left(y_{j}\right) \cdot \eta_{\hat{\mathcal{B}}}^{2}\left(y_{j}\right)}{\xi_{\mathcal{B}}^{4}\left(y_{j}\right)+\eta_{\hat{\mathcal{B}}}^{4}\left(y_{j}\right)+v_{\hat{\mathcal{A}}}^{4}\left(y_{j}\right)} \geq \frac{\eta_{\hat{\mathcal{A}}}^{2}\left(y_{j}\right) \cdot \eta_{\hat{\mathcal{C}}}^{2}\left(y_{j}\right)}{\xi_{\mathcal{C}}^{4}\left(y_{j}\right)+\eta_{\hat{\mathcal{C}}}^{4}\left(y_{j}\right)+v_{\hat{\mathcal{A}}}^{4}\left(y_{j}\right)}, \\
& \frac{v_{\hat{\mathcal{A}}}^{2}\left(y_{j}\right) \cdot v_{\hat{\mathcal{B}}}^{2}\left(y_{j}\right)}{\xi_{\hat{\mathcal{B}}}^{4}\left(y_{j}\right)+\eta_{\mathcal{B}}^{4}\left(y_{j}\right)+v_{\hat{\mathcal{A}}}^{4}\left(y_{j}\right)} \geq \frac{v_{\hat{\mathcal{A}}}^{2}\left(y_{j}\right) \cdot v_{\hat{\mathcal{C}}}^{2}\left(y_{j}\right)}{\xi_{\hat{\mathcal{C}}}^{4}\left(y_{j}\right)+\eta_{\hat{\mathcal{C}}}^{4}\left(y_{j}\right)+v_{\hat{\mathcal{A}}}^{4}\left(y_{j}\right)} .
\end{aligned}
$$

By adding Equations (18)-(20), we have

$$
\begin{gathered}
\frac{\left[\xi_{\hat{\mathcal{A}}}^{2}\left(y_{j}\right) \cdot \xi_{\hat{\mathcal{B}}}^{2}\left(y_{j}\right)+\eta_{\hat{\mathcal{A}}}^{2}\left(y_{j}\right) \cdot \eta_{\hat{\mathcal{B}}}^{2}\left(y_{j}\right)+v_{\hat{\mathcal{A}}}^{2}\left(y_{j}\right) \cdot v_{\hat{\mathcal{B}}}^{2}\left(y_{j}\right)\right]}{\left[\left\{\xi_{\hat{\mathcal{B}}}^{4}\left(y_{j}\right)\right\}+\left\{\eta_{\mathcal{B}}^{4}\left(y_{j}\right)\right\}+\left\{v_{\hat{\mathcal{A}}}^{4}\left(y_{j}\right)\right\}\right]} \geq \frac{\left[\xi_{\hat{\mathcal{A}}}^{2}\left(y_{j}\right) \cdot \xi_{\hat{\mathcal{C}}}^{2}\left(y_{j}\right)+\eta_{\hat{\mathcal{A}}}^{2}\left(y_{j}\right) \cdot \eta_{\hat{\mathcal{C}}}^{2}\left(y_{j}\right)+v_{\hat{\mathcal{A}}}^{2}\left(y_{j}\right) \cdot v_{\hat{\mathcal{C}}}^{2}\left(y_{j}\right)\right]}{\left[\left\{\xi_{\hat{\mathcal{C}}}^{4}\left(y_{j}\right)\right\}+\left\{\eta_{\mathcal{\mathcal { C }}}^{4}\left(y_{j}\right)\right\}+\left\{v_{\hat{\mathcal{A}}}^{4}\left(y_{j}\right)\right\}\right]} \\
\Longrightarrow \frac{\sum_{j=1}^{m}\left[\xi_{\hat{\mathcal{A}}}^{2}\left(y_{j}\right) \cdot \xi_{\hat{\mathcal{B}}}^{2}\left(y_{j}\right)+\eta_{\hat{\mathcal{A}}}^{2}\left(y_{j}\right) \cdot \eta_{\hat{\mathcal{B}}}^{2}\left(y_{j}\right)+v_{\hat{\mathcal{A}}}^{2}\left(y_{j}\right) \cdot v_{\hat{\mathcal{B}}}^{2}\left(y_{j}\right)\right]}{\sum_{j=1}^{m}\left[\left\{\xi_{\hat{\mathcal{B}}}^{4}\left(y_{j}\right)\right\}+\left\{\eta_{\hat{\mathcal{B}}}^{4}\left(y_{j}\right)\right\}+\left\{v_{\hat{\mathcal{A}}}^{4}\left(y_{j}\right)\right\}\right]} \\
\geq \frac{\sum_{j=1}^{m}\left[\xi_{\hat{\mathcal{A}}}^{2}\left(y_{j}\right) \cdot \xi_{\hat{\mathcal{C}}}^{2}\left(y_{j}\right)+\eta_{\hat{\mathcal{A}}}^{2}\left(y_{j}\right) \cdot \eta_{\hat{\mathcal{C}}}^{2}\left(y_{j}\right)+v_{\hat{\mathcal{A}}}^{2}\left(y_{j}\right) \cdot v_{\hat{\mathcal{C}}}^{2}\left(y_{j}\right)\right]}{\sum_{j=1}^{m}\left[\left\{\xi_{\hat{\mathcal{C}}}^{4}\left(y_{j}\right)\right\}+\left\{\eta_{\hat{\mathcal{C}}}^{4}\left(y_{j}\right)\right\}+\left\{v_{\hat{\mathcal{A}}}^{4}\left(y_{j}\right)\right\}\right]} .
\end{gathered}
$$

Hence $\mathcal{S}^{\mathcal{S}}(\hat{\mathcal{A}}, \hat{\mathcal{C}}) \leq \mathcal{S}^{s}(\hat{\mathcal{A}}, \hat{\mathcal{B}})$. Similarly, we can prove $\mathcal{S}^{s}(\hat{\mathcal{A}}, \hat{\mathcal{C}}) \leq \mathcal{S}^{s}(\hat{\mathcal{B}}, \hat{\mathcal{C}})$. and $\hat{\mathcal{B}}$.

Hence from (S1) - (S4), we conclude that $\mathcal{S}^{s}(\hat{\mathcal{A}}, \hat{\mathcal{B}})$ is the similarity measure between $\mathcal{S} \mathcal{F} \mathcal{S} S \hat{\mathcal{A}}$

Definition 12. Two $\mathcal{S} \mathcal{F} \mathcal{S} s \hat{\mathcal{A}}$ and $\hat{\mathcal{B}}$ are called $\simeq^{\alpha}$-similar, denoted as $\hat{\mathcal{A}} \simeq^{\alpha} \hat{\mathcal{B}}$, if and only if $\mathcal{S}^{s}(\hat{\mathcal{A}}, \hat{\mathcal{C}}) \geq \alpha$ for $\alpha \in(0,1)$.

Corollary 1. $\simeq^{\alpha}$ is reflexive and symmetric.

Proof. The reflexive and symmetric part follows from Theorem 1.

The following example shows that the relation $\simeq^{\alpha}$ is not transitive.

Example 2. Let $\hat{\mathcal{Y}}=\left\{y_{1}, y_{2}, y_{3}, y_{4}, y_{5}\right\}$ be the universal set. Let us define $\alpha=0.5$. We consider three $\mathcal{S} \mathcal{F} \mathcal{S}$ s $\hat{\mathcal{A}}, \hat{\mathcal{B}}$ and $\hat{\mathcal{C}}$ in $\hat{\mathcal{Y}}$, which are given as follows:

$$
\begin{aligned}
& \hat{\mathcal{A}}=\left\{(0.8,0.1,0.0) / y_{1},(0.6,0.2,0.1) / y_{2},(0.1,0.1,0.8) / y_{3},(0.6,0.1,0.2) / y_{4},(0.2,0.1,0.6) / y_{5}\right\} \\
& \hat{\mathcal{B}}=\left\{(0.6,0.0,0.4) / y_{1},(0.6,0.2,0.5) / y_{2},(0.4,0.1,0.7) / y_{3},(0.8,0.1,0.3) / y_{4},(0.6,0.1,0.7) / y_{5}\right\} \\
& \hat{\mathcal{C}}=\left\{(0.5,0.3,0.2) / y_{1},(0.3,0.1,0.5) / y_{2},(0.3,0.3,0.4) / y_{3},(0.7,0.1,0.2) / y_{4},(0.6,0.1,0.1) / y_{5}\right\} .
\end{aligned}
$$

Then $\mathcal{S}^{s}(\hat{\mathcal{A}}, \hat{\mathcal{B}})=0.59636>0.5$ and $\mathcal{S}^{s}(\hat{\mathcal{B}}, \hat{\mathcal{C}})=0.519811>0.5$, but $\mathcal{S}^{s}(\hat{\mathcal{A}}, \hat{\mathcal{C}})=0.322488<0.5$. Hence the relation $\simeq^{\alpha}$ is not transitive. 
Sometimes the alternatives under observations are not of equal importance, therefore, we defines weights of alternatives to signify their importance and defines weighted similarity measures between $\mathcal{S} \mathcal{F} \mathcal{S}$ s.

Definition 13. For two $\mathcal{S} \mathcal{F} \mathcal{S}$ s $\hat{\mathcal{A}}$ and $\hat{\mathcal{B}}$ in $\hat{\mathcal{Y}}$, a new weighted similarity measure is defined between $\hat{\mathcal{A}}$ and $\hat{\mathcal{B}}$ as follows:

$$
\mathcal{S}_{\omega}^{s}(\hat{\mathcal{A}}, \hat{\mathcal{B}})=\frac{\sum_{j=1}^{m} \omega_{j}\left[\xi_{\hat{\mathcal{A}}}^{2}\left(y_{j}\right) \cdot \xi_{\hat{\mathcal{B}}}^{2}\left(y_{j}\right)+\eta_{\hat{\mathcal{A}}}^{2}\left(y_{j}\right) \cdot \eta_{\hat{\mathcal{B}}}^{2}\left(y_{j}\right)+v_{\hat{\mathcal{A}}}^{2}\left(y_{j}\right) \cdot v_{\hat{\mathcal{B}}}^{2}\left(y_{j}\right)\right]}{\sum_{j=1}^{m}\left[\left\{\xi_{\hat{\mathcal{A}}}^{4}\left(y_{j}\right) \vee \xi_{\hat{\mathcal{B}}}^{4}\left(y_{j}\right)\right\}+\left\{\eta_{\hat{\mathcal{A}}}^{4}\left(y_{j}\right) \vee \eta_{\hat{\mathcal{B}}}^{4}\left(y_{j}\right)\right\}+\left\{v_{\hat{\mathcal{A}}}^{4}\left(y_{j}\right) \vee v_{\hat{\mathcal{B}}}^{4}\left(y_{j}\right)\right\}\right]} /\left(\sum_{j=1}^{m} \omega_{j}\right),
$$

where $\omega_{j} \in[0,1]$ are the weights of alternatives, but not all zero, $1 \leq j \leq m$. If $\sum_{j=1}^{m} \omega_{j}=1$, then we have

$$
\mathcal{S}_{\omega}^{s}(\hat{\mathcal{A}}, \hat{\mathcal{B}})=\frac{\sum_{j=1}^{m} \omega_{j}\left[\xi_{\hat{\mathcal{A}}}^{2}\left(y_{j}\right) \cdot \xi_{\hat{\mathcal{B}}}^{2}\left(y_{j}\right)+\eta_{\hat{\mathcal{A}}}^{2}\left(y_{j}\right) \cdot \eta_{\hat{\mathcal{B}}}^{2}\left(y_{j}\right)+v_{\hat{\mathcal{A}}}^{2}\left(y_{j}\right) \cdot v_{\hat{\mathcal{B}}}^{2}\left(y_{j}\right)\right]}{\sum_{j=1}^{m}\left[\left\{\xi_{\hat{\mathcal{A}}}^{4}\left(y_{j}\right) \vee \xi_{\hat{\mathcal{B}}}^{4}\left(y_{j}\right)\right\}+\left\{\eta_{\hat{\mathcal{A}}}^{4}\left(y_{j}\right) \vee \eta_{\hat{\mathcal{B}}}^{4}\left(y_{j}\right)\right\}+\left\{v_{\hat{\mathcal{A}}}^{4}\left(y_{j}\right) \vee v_{\hat{\mathcal{B}}}^{4}\left(y_{j}\right)\right\}\right]} .
$$

Example 3. In Example 1, consider 0.8, 0.5, 0.6, 0.7 and 0.6 be the weights of $y_{1}, y_{2}, y_{3}, y_{4}$ and $y_{5}$, respectively. Then

$$
\mathcal{S}_{\omega}^{\varsigma}(\hat{\mathcal{A}}, \hat{\mathcal{B}})=\frac{0.44678}{1.6585}=0.269388
$$

Theorem 2. $\mathcal{S}_{\omega}^{s}(\hat{\mathcal{A}}, \hat{\mathcal{B}})$ is the similarity measure between two $\mathcal{S} \mathcal{F} \mathcal{S} s \hat{\mathcal{A}}$ and $\hat{\mathcal{B}}$ in $\hat{\mathcal{Y}}$.

Proof. The proof is similar to the proof of Theorem 1.

On the basis of new similarity measure $\mathcal{S}^{s}$, we define distance measures for $\mathcal{S} \mathcal{F} \mathcal{S}$.

Definition 14. For two $\mathcal{S} \mathcal{F} \mathcal{S} S \hat{\mathcal{A}}$ and $\hat{\mathcal{B}}$ in $\hat{\mathcal{Y}}$, a new distance measures is defined between $\hat{\mathcal{A}}$ and $\hat{\mathcal{B}}$ as follows:

$$
\mathcal{D}^{s}(\hat{\mathcal{A}}, \hat{\mathcal{B}})=1-\frac{\sum_{j=1}^{m}\left[\xi_{\hat{\mathcal{A}}}^{2}\left(y_{j}\right) \cdot \xi_{\hat{\mathcal{B}}}^{2}\left(y_{j}\right)+\eta_{\hat{\mathcal{A}}}^{2}\left(y_{j}\right) \cdot \eta_{\hat{\mathcal{B}}}^{2}\left(y_{j}\right)+v_{\hat{\mathcal{A}}}^{2}\left(y_{j}\right) \cdot v_{\hat{\mathcal{B}}}^{2}\left(y_{j}\right)\right]}{\sum_{j=1}^{m}\left[\left\{\xi_{\hat{\mathcal{A}}}^{4}\left(y_{j}\right) \vee \xi_{\hat{\mathcal{B}}}^{4}\left(y_{j}\right)\right\}+\left\{\eta_{\hat{\mathcal{A}}}^{4}\left(y_{j}\right) \vee \eta_{\hat{\mathcal{B}}}^{4}\left(y_{j}\right)\right\}+\left\{v_{\hat{\mathcal{A}}}^{4}\left(y_{j}\right) \vee v_{\hat{\mathcal{B}}}^{4}\left(y_{j}\right)\right\}\right]} .
$$

Definition 15. For two $\mathcal{S} \mathcal{F} \mathcal{S} S \hat{\mathcal{A}}$ and $\hat{\mathcal{B}}$ in $\hat{\mathcal{Y}}$, a new weighted distance measure is defined between $\hat{\mathcal{A}}$ and $\hat{\mathcal{B}}$ as follows:

$$
\mathcal{D}_{\omega}^{s}(\hat{\mathcal{A}}, \hat{\mathcal{B}})=1-\frac{\sum_{j=1}^{m} \omega_{j}\left[\xi_{\hat{\mathcal{A}}}^{2}\left(y_{j}\right) \cdot \xi_{\hat{\mathcal{B}}}^{2}\left(y_{j}\right)+\eta_{\hat{\mathcal{A}}}^{2}\left(y_{j}\right) \cdot \eta_{\hat{\mathcal{B}}}^{2}\left(y_{j}\right)+v_{\hat{\mathcal{A}}}^{2}\left(y_{j}\right) \cdot v_{\hat{\mathcal{B}}}^{2}\left(y_{j}\right)\right]}{\sum_{j=1}^{m}\left[\left\{\xi_{\hat{\mathcal{A}}}^{4}\left(y_{j}\right) \vee \xi_{\hat{\mathcal{B}}}^{4}\left(y_{j}\right)\right\}+\left\{\eta_{\hat{\mathcal{A}}}^{4}\left(y_{j}\right) \vee \eta_{\mathcal{B}}^{4}\left(y_{j}\right)\right\}+\left\{v_{\hat{\mathcal{A}}}^{4}\left(y_{j}\right) \vee v_{\hat{\mathcal{B}}}^{4}\left(y_{j}\right)\right\}\right]} /\left(\sum_{j=1}^{m} \omega_{j}\right),
$$

where $\omega_{j} \in[0,1]$ are the weights of alternatives, but not all zero, $1 \leq j \leq m$. If $\sum_{j=1}^{m} \omega_{j}=1$, then we have

$$
\mathcal{D}_{\omega}^{s}(\hat{\mathcal{A}}, \hat{\mathcal{B}})=1-\frac{\sum_{j=1}^{m} \omega_{j}\left[\xi_{\hat{\mathcal{A}}}^{2}\left(y_{j}\right) \cdot \xi_{\hat{\mathcal{B}}}^{2}\left(y_{j}\right)+\eta_{\hat{\mathcal{A}}}^{2}\left(y_{j}\right) \cdot \eta_{\hat{\mathcal{B}}}^{2}\left(y_{j}\right)+v_{\hat{\mathcal{A}}}^{2}\left(y_{j}\right) \cdot v_{\hat{\mathcal{B}}}^{2}\left(y_{j}\right)\right]}{\sum_{j=1}^{m}\left[\left\{\xi_{\hat{\mathcal{A}}}^{4}\left(y_{j}\right) \vee \xi_{\hat{\mathcal{B}}}^{4}\left(y_{j}\right)\right\}+\left\{\eta_{\hat{\mathcal{A}}}^{4}\left(y_{j}\right) \vee \eta_{\hat{\mathcal{B}}}^{4}\left(y_{j}\right)\right\}+\left\{v_{\hat{\mathcal{A}}}^{4}\left(y_{j}\right) \vee v_{\hat{\mathcal{B}}}^{4}\left(y_{j}\right)\right\}\right]} .
$$

Theorem 3. $\mathcal{D}^{s}$ and $\mathcal{D}_{\omega}^{s}$ are the distance measures between $\mathcal{S} \mathcal{F} \mathcal{S}$ s.

Proof. The proof is similar to the proof of Theorem 1. 


\section{Applications in Pattern Recognition and Counter Examples}

In this section, we provide some counter examples for already proposed similarity measures in the literature in pattern recognition. We have seen that the already proposed measures cannot classify the unknown pattern while set theoretic similarity measure classify the unknown pattern, which shows that our proposed similarity measure is applicable in pattern recognition problems.

Example 4. In this example, we have seen that the second condition of Definition $10\left(S_{2}\right)$ is not satisfied for cosine similarity measure $\mathcal{S}_{c}^{1}$ (Definition 4), i.e., if $\hat{\mathcal{A}}=\left\{(a, a, a) / y_{j} \mid y_{j} \in \hat{\mathcal{Y}}, 1 \leq j \leq m\right\}$ and $\hat{\mathcal{B}}=\left\{(b, b, b) / y_{j} \mid y_{j} \in \hat{\mathcal{Y}}, 1 \leq j \leq m\right\}$ are two $\mathcal{S} \mathcal{F} \mathcal{S}$ s in $\hat{\mathcal{Y}}$ with $0 \leq a, b \leq 1,0 \leq a^{2}+a^{2}+a^{2} \leq 1$, $0 \leq b^{2}+b^{2}+b^{2} \leq 1$ and $a \neq b$, then $\hat{\mathcal{A}} \neq \hat{\mathcal{B}}$. However, $\mathcal{S}_{c}^{1}(\hat{\mathcal{A}}, \hat{\mathcal{B}})=1$.

For example, let $\hat{\mathcal{Y}}=\left\{y_{1}, y_{2}, y_{3}\right\}$ and $\mathcal{S} \mathcal{F} \mathcal{S}$ s in $\hat{\mathcal{Y}}$ are

$$
\begin{aligned}
\hat{\mathcal{A}} & =\left\{(0.50,0.50,0.50) / y_{1},(0.30,0.30,0.30) / y_{2},(0.40,0.40,0.40) / y_{3}\right\} \text { and } \\
\hat{\mathcal{B}} & =\left\{(0.41,0.41,0.41) / y_{1},(0.27,0.27,0.27) / y_{2},(0.33,0.33,0.33) / y_{3}\right\} .
\end{aligned}
$$

Clearly, $\hat{\mathcal{A}} \neq \hat{\mathcal{B}}$ but $\mathcal{S}_{\mathcal{c}}^{1}(\hat{\mathcal{A}}, \hat{\mathcal{B}})=1$. Hence $\mathcal{S}_{c}^{1}$ is not effective for these cases and not reliable to find the similarity measure between $\mathcal{S} \mathcal{F} \mathcal{S}$ s. However, when we find the similarity measure by using $\mathcal{S}^{s}$, we get $\mathcal{S}^{S}(\hat{\mathcal{A}}, \hat{\mathcal{B}})=0.686175$.

Example 5. Let $Q_{1}$ and $Q_{2}$ be two known patterns with class labels $Z_{1}$ and $Z_{2}$, respectively, are given. The $\mathcal{S} \mathcal{F} \mathcal{S}$ s are used to represents the patterns in $\hat{\mathcal{Y}}=\left\{y_{1}, y_{2}, y_{3}\right\}$ as follows:

$$
\begin{aligned}
& Q_{1}=\left\{(0.7,0.5,0.3) / y_{1},(0.4,0.3,0.5) / y_{2},(0.6,0.4,0.3) / y_{3}\right\} \\
& Q_{2}=\left\{(0.5,0.7,0.3) / y_{1},(0.3,0.4,0.5) / y_{2},(0.4,0.6,0.3) / y_{3}\right\} .
\end{aligned}
$$

$P$ is the unknown pattern which is given as follows:

$$
P=\left\{(0.5,0.4,0.4) / y_{1},(0.4,0.6,0.5) / y_{2},(0.6,0.7,0.3) / y_{3}\right\}
$$

Our aim is to find out the class of unknown pattern $P$ belongs to. However, when we use cosine similarity measure $\mathcal{S}_{c}^{3}$ (Definition 5), we get the same similarity measure i.e., $\mathcal{S}_{c}^{3}\left(P, Q_{1}\right)=\mathcal{S}_{c}^{3}\left(P, Q_{2}\right)=0.965086$. Furthermore, when we use the cotangent similarity measure $\mathcal{S}_{c}^{5}$ (Definition 6), we get the same similarity measures i.e., $\mathcal{S}_{c}^{5}\left(P, Q_{1}\right)=\mathcal{S}_{c}^{5}\left(P, Q_{2}\right)=0.767857$. Hence in this case we cannot decide the class of unknown pattern $P$ by using $\mathcal{S}_{c}^{3}$ and $\mathcal{S}_{c}^{5}$. However, when we find the similarity measure by using $\mathcal{S}^{s}$, we get $\mathcal{S}^{S}\left(P, Q_{1}\right)=0.555916$ and $\mathcal{S}^{s}\left(P, Q_{2}\right)=0.575836$. Since $\mathcal{S}^{S}\left(P, Q_{2}\right)>\mathcal{S}^{S}\left(P, Q_{1}\right)$, therefore, the unknown pattern $P$ belongs to class $Z_{2}$.

Example 6. Let $Q_{1}$ and $Q_{2}$ be two known patterns with class labels $Z_{1}$ and $Z_{2}$, respectively, are given. The $\mathcal{S} \mathcal{F} \mathcal{S}$ s are used to represents the patterns in $\hat{\mathcal{Y}}=\left\{y_{1}, y_{2}, y_{3}\right\}$ as follows:

$$
\begin{aligned}
& Q_{1}=\left\{(0.5,0.7,0.3) / y_{1},(0.3,0.8,0.4) / y_{2},(0.6,0.3,0.1) / y_{3}\right\} \\
& Q_{2}=\left\{(0.6,0.7,0.3) / y_{1},(0.8,0.4,0.4) / y_{2},(0.6,0.4,0.2) / y_{3}\right\} .
\end{aligned}
$$

$P$ is the unknown pattern which is given as follows:

$$
P=\left\{(0.6,0.4,0.3) / y_{1},(0.5,0.5,0.4) / y_{2},(0.7,0.4,0.2) / y_{3}\right\}
$$

Our aim is to find out the class of unknown pattern $P$ belongs to. However, when we use cosine similarity measure $\mathcal{S}_{c}^{2}$ (Definition 5), we get the same similarity measure i.e., $\mathcal{S}_{c}^{2}\left(P, Q_{1}\right)=\mathcal{S}_{c}^{2}\left(P, Q_{2}\right)=0.888668$. Furthermore, when we use the cotangent similarity measure $\mathcal{S}_{c}^{4}$ (Definition 6), we get the same similarity measures i.e., $\mathcal{S}_{c}^{4}\left(P, Q_{1}\right)=\mathcal{S}_{c}^{4}\left(P, Q_{2}\right)=0.638144$. Hence in this case we cannot decide the class of 
unknown pattern $P$ by using $\mathcal{S}_{c}^{2}$ and $\mathcal{S}_{c}^{4}$. However, when we find the similarity measure by using $\mathcal{S}^{s}$, we get $\mathcal{S}^{S}\left(P, Q_{1}\right)=0.50385$ and $\mathcal{S}^{S}\left(P, Q_{2}\right)=0.564666$. Since $\mathcal{S}^{S}\left(P, Q_{2}\right)>\mathcal{S}^{S}\left(P, Q_{1}\right)$, therefore, the unknown pattern $P$ belongs to class $Z_{2}$.

Example 7. Let $Q_{1}$ and $Q_{2}$ be two known patterns with class labels $Z_{1}$ and $Z_{2}$, respectively, are given. The $\mathcal{S} \mathcal{F} \mathcal{S}$ s are used to represents the patterns in $\hat{\mathcal{Y}}=\left\{y_{1}, y_{2}, y_{3}\right\}$ as follows:

$$
\begin{aligned}
& Q_{1}=\left\{(0.5,0.7,0.3) / y_{1},(0.3,0.8,0.4) / y_{2},(0.5,0.6,0.4) / y_{3}\right\} \\
& Q_{2}=\left\{(0.6,0.7,0.3) / y_{1},(0.8,0.4,0.4) / y_{2},(0.6,0.5,0.4) / y_{3}\right\} .
\end{aligned}
$$

$P$ is the unknown pattern which is given as follows:

$$
P=\left\{(0.6,0.4,0.3) / y_{1},(0.5,0.5,0.4) / y_{2},(0.6,0.5,0.2) / y_{3}\right\}
$$

Our aim is to find out the class of unknown pattern $P$ belongs to. However, when we use cosine similarity measure $\mathcal{S}_{c}^{6}$ (Definition 7), we get the same similarity measure i.e., $\mathcal{S}_{c}^{6}\left(P, Q_{1}\right)=\mathcal{S}_{c}^{6}\left(P, Q_{2}\right)=0.889689$. Furthermore, when we use the cotangent similarity measure $\mathcal{S}_{c}^{8}$ (Definition 8), we get the same similarity measures i.e., $\mathcal{S}_{c}^{8}\left(P, Q_{1}\right)=\mathcal{S}_{c}^{8}\left(P, Q_{2}\right)=0.642526$. Hence in this case we cannot decide the class of unknown pattern $P$ by using $\mathcal{S}_{c}^{6}$ and $\mathcal{S}_{c}^{8}$. However, when we find the similarity measure by using $\mathcal{S}^{s}$, we get $\mathcal{S}^{s}\left(P, Q_{1}\right)=0.492114$ and $\mathcal{S}^{s}\left(P, Q_{2}\right)=0.58562$. Since $\mathcal{S}^{s}\left(P, Q_{2}\right)>\mathcal{S}^{s}\left(P, Q_{1}\right)$, therefore, the unknown pattern $P$ belongs to class $Z_{2}$.

Example 8. Let $Q_{1}$ and $Q_{2}$ be two known patterns with class labels $Z_{1}$ and $Z_{2}$, respectively, are given. The $\mathcal{S} \mathcal{F} \mathcal{S}$ s are used to represents the patterns in $\hat{\mathcal{Y}}=\left\{y_{1}, y_{2}, y_{3}\right\}$ as follows:

$$
\begin{aligned}
& Q_{1}=\left\{(0.5,0.7,0.3) / y_{1},(0.3,0.8,0.4) / y_{2},(0.5,0.6,0.4) / y_{3}\right\} \\
& Q_{2}=\left\{(0.6,0.7,0.3) / y_{1},(0.8,0.4,0.4) / y_{2},(0.6,0.5,0.4) / y_{3}\right\} .
\end{aligned}
$$

$P$ is the unknown pattern which is given as follows:

$$
P=\left\{(0.6,0.4,0.3) / y_{1},(0.5,0.5,0.4) / y_{2},(0.5,0.5,0.2) / y_{3}\right\}
$$

Our aim is to find out the class of unknown pattern $P$ belongs to. However, when we use cosine similarity measure $\mathcal{S}_{c}^{7}$ (Definition 7), we get the same similarity measure i.e., $\mathcal{S}_{c}^{7}\left(P, Q_{1}\right)=\mathcal{S}_{c}^{7}\left(P, Q_{2}\right)=0.874075$. Furthermore, when we use the cotangent similarity measure $\mathcal{S}_{c}^{9}$ (Definition 8), we get the same similarity measures i.e., $\mathcal{S}_{c}^{9}\left(P, Q_{1}\right)=\mathcal{S}_{c}^{9}\left(P, Q_{2}\right)=0.597149$. Hence in this case we cannot decide the class of unknown pattern $P$ by using $\mathcal{S}_{c}^{7}$ and $\mathcal{S}_{c}^{9}$. However, when we find the similarity measure by using $\mathcal{S}^{s}$, we get $\mathcal{S}^{s}\left(P, Q_{1}\right)=0.497164$ and $\mathcal{S}^{s}\left(P, Q_{2}\right)=0.549396$. Since $\mathcal{S}^{s}\left(P, Q_{2}\right)>\mathcal{S}^{s}\left(P, Q_{1}\right)$, therefore, the unknown pattern $P$ belongs to class $Z_{2}$.

\section{Selection of Mega Projects in Underdeveloping Countries}

The megaprojects are characterized by vast complexity (especially in organizational terms), large investment commitment, long-lasting impact on the economy, the environment, and society. So it is important to choose the best method for the selection of mega projects for under developing countries. Because it affects the lives of millions of peoples, take much time to develop and build, involve multiple public and private stakeholders, and have a long-lasting impact on the economy, the environment, and society. As we have seen that the proposed similarity measures have counter-intuitive cases for Examples 4-8. Therefore, the selection of mega projects for under developing countries is done by the proposed similarity measure.

It is important for under developing countries to select upcoming mega projects on priority which has less effect on their economy, environment, less maintenance cost has long term benefits, fewer 
peoples effects from that project and generate high revenue. For example, a country has to start the mega project and they get a loan from the world bank so the country has to think before spending the money because they have to refund after some time. The government has five projects in his focus like 1 million house construction, dam construction, orange metro train, invest in industry and power sector. This set can be represented as $U$ and the elements of $U$ represented as $E_{i}, 1 \leq i \leq 5$, that is

$U=\{1$ million house construction, dam construction, orange metro train, invest in industry,

power sector $\}$.

To select the project on a priority basis, there are some parameters selected by experts from different fields to check the importance of projects like long term benefits, time, impact, revenue generated, costs and short term benefits. We represents this criteria as a set $W$ and the elements of $W$ represented as $e_{j}, 1 \leq j \leq 6$, that is

$$
W=\{\text { long term benefits, time, impact, revenue generated, cost, short term benefits }\} .
$$

We apply proposed technique for selecting upcoming mega projects on priority basis which is the classical multi attribute decision making problem. The weight vector for each attribute $e_{j}, j \in\{1,2, \ldots, 6\}$ is $\hat{\omega}=(0.12,0.25,0.09,0.16,0.20,0.18)^{T}$. All the data collected in spherical fuzzy information is summarized in Table 1 . In Table 1 , we have seen that for each mega project $E_{i}, i \in\{1,2, \ldots, 5\}$, experts interpret their evaluation in the form of $S F V S$ corresponding to each attribute (criteria).

To apply the proposed method, we calculate the ideal alternative (mega project) $E^{+}$from given data as follows $\mu_{j}^{+}=\operatorname{Max}_{i}\left\{\mu_{i}\right\}, 1 \leq j \leq 6,1 \leq i \leq 5, \eta_{j}^{+}=\operatorname{Max}_{i}\left\{\eta_{i}\right\}, 1 \leq j \leq 6,1 \leq i \leq 5$ and $v_{j}^{+}=\operatorname{Max}_{i}\left\{v_{i}\right\}, 1 \leq j \leq 6,1 \leq i \leq 5$. Then the similarity measures between each alternative and ideal alternative are calculated. Heigh values of similarity measure more closer to the ideal alternative. In this case, the ideal alternative is

$$
\begin{array}{r}
E^{+}=\{(0.91,0.03,0.02),(0.89,0.08,0.03),(0.42,0.35,0.05),(0.73,0.15,0.02),(0.52,0.31,0.05) \\
(0.91,0.03,0.05)\}
\end{array}
$$

Then the similarity measures $\mathcal{S}^{s}$ between between each alternative $E_{i}$ and ideal alternative $E^{+}$ are calculated. The details of similarity measures presented in Table 2 and the ranking of alternatives (mega projects) is given as follows:

$$
E_{5} \succ E_{4} \succ E_{1} \succ E_{2} \succ E_{3} \text {. }
$$

The comparison between the already proposed similarity measures and proposed similarity measure is presented in Table 2.

Table 1. Data Table.

\begin{tabular}{cccccc}
\hline & $\boldsymbol{E}_{\mathbf{1}}$ & $\boldsymbol{E}_{\mathbf{2}}$ & $\boldsymbol{E}_{\mathbf{3}}$ & $\boldsymbol{E}_{\mathbf{4}}$ & $\boldsymbol{E}_{\mathbf{5}}$ \\
\hline$e_{1}$ & $(0.53,0.33,0.09)$ & $(0.73,0.12,0.08)$ & $(0.91,0.03,0.02)$ & $(0.85,0.09,0.05)$ & $(0.90,0.05,0.02)$ \\
$e_{2}$ & $(0.89,0.08,0.03)$ & $(0.13,0.64,0.21)$ & $(0.07,0.09,0.05)$ & $(0.74,0.16,0.10)$ & $(0.68,0.08,0.21)$ \\
$e_{3}$ & $(0.42,0.35,0.18)$ & $(0.03,0.82,0.13)$ & $(0.04,0.85,0.10)$ & $(0.02,0.89,0.05)$ & $(0.05,0.87,0.06)$ \\
$e_{4}$ & $(0.08,0.89,0.02)$ & $(0.73,0.15,0.08)$ & $(0.68,0.26,0.06)$ & $(0.08,0.84,0.06)$ & $(0.13,0.75,0.09)$ \\
$e_{5}$ & $(0.33,0.51,0.12)$ & $(0.52,0.31,0.16)$ & $(0.15,0.76,0.07)$ & $(0.16,0.71,0.05)$ & $(0.15,0.73,0.08)$ \\
$e_{6}$ & $(0.17,0.53,0.13)$ & $(0.51,0.24,0.21)$ & $(0.31,0.39,0.25)$ & $(0.81,0.15,0.09)$ & $(0.91,0.03,0.05)$ \\
\hline
\end{tabular}


Table 2. Similarity Measures.

\begin{tabular}{ccccccc}
\hline Similarity Measures & $\mathcal{S}\left(E^{+}, E_{\mathbf{1}}\right)$ & $\mathcal{S}\left(E^{+}, E_{\mathbf{2}}\right)$ & $\mathcal{S}\left(E^{+}, E_{\mathbf{3}}\right)$ & $\mathcal{S}\left(E^{+}, E_{\mathbf{4}}\right)$ & $\mathcal{S}\left(E^{+}, E_{5}\right)$ & Ranking \\
\hline $\mathcal{S}^{S}$ & 0.0650871 & 0.054608 & 0.048139 & 0.079880 & 0.089495 & $E_{5} \succ E_{4} \succ E_{1} \succ E_{2} \succ E_{3}$ \\
$\mathcal{S}_{c}^{1}$ & 0.102027 & 0.119422 & 0.103822 & 0.114281 & 0.114483 & $E_{2} \succ E_{5} \succ E_{4} \succ E_{3} \succ E_{1}$ \\
$\mathcal{S}_{c}^{2}$ & 0.120687 & 0.120848 & 0.105494 & 0.13416 & 0.137768 & $E_{5} \succ E_{4} \succ E_{2} \succ E_{1} \succ E_{3}$ \\
$\mathcal{S}_{c}^{3}$ & 0.139783 & 0.141826 & 0.142814 & 0.146582 & 0.148426 & $E_{5} \succ E_{4} \succ E_{3} \succ E_{2} \succ E_{1}$ \\
$\mathcal{S}_{c}^{4}$ & 0.098745 & 0.094479 & 0.076557 & 0.095334 & 0.103948 & $E_{5} \succ E_{1} \succ E_{4} \succ E_{2} \succ E_{3}$ \\
$\mathcal{S}_{c}^{5}$ & 0.112116 & 0.113426 & 0.104831 & 0.112798 & 0.117358 & $E_{5} \succ E_{2} \succ E_{4} \succ E_{1} \succ E_{3}$ \\
$\mathcal{S}_{C}^{6}$ & 0.120687 & 0.120848 & 0.105494 & 0.13416 & 0.137768 & $E_{5} \succ E_{4} \succ E_{2} \succ E_{1} \succ E_{3}$ \\
$\mathcal{S}_{c}^{7}$ & 0.120523 & 0.120588 & 0.105265 & 0.134042 & 0.137374 & $E_{5} \succ E_{4} \succ E_{2} \succ E_{1} \succ E_{3}$ \\
$\mathcal{S}_{c}^{8}$ & 0.098745 & 0.094479 & 0.0765566 & 0.0953338 & 0.103948 & $E_{5} \succ E_{1} \succ E_{4} \succ E_{2} \succ E_{3}$ \\
$\mathcal{S}_{c}^{9}$ & 0.098252 & 0.0942871 & 0.0763846 & 0.0952625 & 0.103626 & $E_{5} \succ E_{1} \succ E_{4} \succ E_{2} \succ E_{3}$ \\
\hline
\end{tabular}

\section{Comparison Analysis}

A comparison between new proposed similarity measure and already proposed similarity measure for $\mathcal{S} \mathcal{F} \mathcal{S}_{S}$ is conducted to illustrate the superiority of the new similarity measure.

We have seen from Example 4 that the second condition of Definition $10\left(S_{2}\right)$ is not satisfied for cosine similarity measure $\mathcal{S}_{c}^{1}$, i.e., $\hat{\mathcal{S}}(\hat{\mathcal{A}}, \hat{\mathcal{B}})=1$ even $\hat{\mathcal{A}} \neq \hat{\mathcal{B}}$. Furthermore, we provide a general criteria when second condition of Definition $10\left(S_{2}\right)$ is not satisfied for cosine similarity measure $\mathcal{S}_{c}^{1}$. In Example 5, we have seen that the $\mathcal{S}_{c}^{3}$ and $\mathcal{S}_{c}^{5}$ can not classify the unknown pattern from known pattern. In Example 6, we have seen that the $\mathcal{S}_{c}^{2}$ and $\mathcal{S}_{c}^{4}$ can not classify the unknown pattern from known pattern. In Example 7, we have seen that the $\mathcal{S}_{c}^{6}$ and $\mathcal{S}_{c}^{8}$ can not classify the unknown pattern from known pattern. In Example 8, we have seen that the $\mathcal{S}_{c}^{7}$ and $\mathcal{S}_{c}^{9}$ can not classify the unknown pattern from known pattern.

However, in all Examples 4-8, the new similarity measure $\mathcal{S}^{\mathcal{S}}$ classify the unknown pattern and hence successfully applicable to the pattern recognition problems. In Section $5, \mathcal{S}^{\mathcal{S}}$ applied successfully to selecting the mega projects for under developing countries.

From Table 3, we have seen that for different special cases, the already proposed similarity measures are not illegible for classification of unknown pattern but $\mathcal{S}^{s}$ applied successfully. For cases 1 and 2, the similarity measures $\mathcal{S}_{c}^{3}, \mathcal{S}_{c}^{5}, \mathcal{S}_{c}^{7}$ and $\mathcal{S}_{c}^{9}$ provide counter-intuitive cases. The similarity measures $\mathcal{S}_{c}^{2}, \mathcal{S}_{c}^{4}, \mathcal{S}_{c}^{6}$ and $\mathcal{S}_{c}^{8}$ provide counter-intuitive cases for 3 and 4 cases. The second axiom of similarity measure for $\mathcal{S}_{c}^{1}$ (Definition 4 ) is not satisfied for case 5 . As we have seen in Example 4, that if we have membership, neutral and non-membership degrees for a set are equal but different from another set which has also same membership degrees, then the $\mathcal{S}_{c}^{1}$ has result 1 . This is inconsistent with the definition of a similarity measure.

Table 3. Comparison Table.

\begin{tabular}{cccccc}
\hline Similarity Measures/Cases & $\mathbf{1}$ & $\mathbf{2}$ & $\mathbf{3}$ & $\mathbf{4}$ & $\mathbf{5}$ \\
\hline$\hat{\mathcal{A}}$ & $(0.5,0.4,0.6)$ & $(0.5,0.4,0.6)$ & $(0.6,0.4,0.3)$ & $(0.6,0.4,0.3)$ & $(0.5,0.5,0.5)$ \\
$\hat{\mathcal{B}}$ & $(0.7,0.5,0.3)$ & $(0.5,0.7,0.3)$ & $(0.5,0.7,0.4)$ & $(0.6,0.7,0.3)$ & $(0.4,0.4,0.4)$ \\
$\mathcal{S}_{c}^{1}$ & 0.749397 & 0.666344 & 0.789612 & 0.870023 & $\mathbf{1 . 0 0}$ \\
$\mathcal{S}_{c}^{2}$ & 0.303801 & 0.289544 & $\mathbf{0 . 2 8 9 5 4 4}$ & $\mathbf{0 . 2 8 9 5 4 4}$ & 0.330008 \\
$\mathcal{S}_{c}^{3}$ & $\mathbf{0 . 2 9 7 0 0 2}$ & $\mathbf{0 . 2 9 7 0 0 2}$ & 0.306948 & 0.322200 & 0.325867 \\
$\mathcal{S}_{c}^{4}$ & 0.215231 & 0.193615 & $\mathbf{0 . 1 9 3 6 1 5}$ & $\mathbf{0 . 1 9 3 6 1 5}$ & 0.289252 \\
$\mathcal{S}_{c}^{5}$ & $\mathbf{0 . 2 0 4 2 6 7}$ & $\mathbf{0 . 2 0 4 2 6 7}$ & 0.220838 & 0.256470 & 0.269206 \\
$\mathcal{S}_{c}^{6}$ & 0.303801 & 0.289544 & $\mathbf{0 . 2 8 9 5 4 4}$ & $\mathbf{0 . 2 8 9 5 4 4}$ & 0.303801 \\
$\mathcal{S}_{c}^{7}$ & $\mathbf{0 . 2 8 9 5 4 4}$ & $\mathbf{0 . 2 8 9 5 4 4}$ & 0.269672 & 0.289544 & 0.303801 \\
$\mathcal{S}_{c}^{8}$ & 0.215231 & 0.193615 & $\mathbf{0 . 1 9 3 6 1 5}$ & $\mathbf{0 . 1 9 3 6 1 5}$ & 0.215231 \\
$\mathcal{S}_{c}^{9}$ & $\mathbf{0 . 1 9 3 6 1 5}$ & $\mathbf{0 . 1 9 3 6 1 5}$ & 0.169842 & 0.193615 & 0.215231 \\
$\mathcal{S}^{S}$ & 0.450949 & 0.400972 & 0.462434 & 0.571996 & 0.640000 \\
\hline
\end{tabular}




\section{Conclusions}

In this paper, we have defined new similarity measures for $\mathcal{S F} \mathcal{S}$ s called set theoretic similarity measures. We define set theoretic similarity measure, weighted set theoretic similarity measure, set theoretic distance and weighted set theoretic distance measures and provide their proofs in this paper. We discuss some special cases (Examples 4-8) where already proposed similarity measure fails to classify the unknown pattern while the proposed similarity measure successfully applied to the pattern recognition problems. Furthermore, $\mathcal{S}^{s}$ applied successfully to selecting the mega projects for under developing countries.

In the future direction, we will apply the set theoretic similarity measure to data mining, medical diagnosis, decision making, complex group decision making, linguistic summarization risk analysis, pattern recognition, color image retrieval, histogram comparison and image processing.

Author Contributions: All authors contributed equally in this research paper. All authors have read and agreed to the published version of the manuscript.

Funding: Petchra Pra Jom Klao Ph.D. Research Scholarship from King Mongkut's University of Technology Thonburi (KMUTT) and Theoretical and Computational Science (TaCS) Center. Moreover, Poom Kumam was supported by the Thailand Research Fund and the King Mongkut's University of Technology Thonburi under the TRF Research Scholar Grant No. RSA6080047. Moreover, this research work was financially supported by the Rajamangala University of Technology Thanyaburi (RMUTTT) (Grant No. NSF62D0604).

Acknowledgments: This project was supported by Center of Excellence in Theoretical and Computational Science (TaCS-CoE), KMUTT. The first author was supported by the "Petchra Pra Jom Klao Ph.D. Research Scholarship from King Mongkut's University of Technology Thonburi”.

Conflicts of Interest: The authors declare no conflict of interest.

\section{Abbreviations}

The following abbreviations are used in this manuscript:

IFS Intuitionistic Fuzzy Set

$\mathcal{P F S}$ Picture Fuzzy Set

$\mathcal{S F S}$ Spherical Fuzzy Set

\section{References}

1. Zadeh, L.A. Fuzzy sets. Inf. Contr. 1965, 8, 338-353. [CrossRef]

2. Atanassov, K.T. Intuitionistic fuzzy sets. Fuzzy Sets Syst. 1986, 20, 87-96. [CrossRef]

3. Atanassov, K.T. Interval valued intuitionistic fuzzy sets. Fuzzy Sets Syst. 1989, 31, 343-349. [CrossRef]

4. Molodtsov, Soft set theory-first results. Comput. Math. Appl. 1999, 37, 19-31. [CrossRef]

5. Maji, P.K.; Biswas, R.; Roy, A.R. Fuzzy soft sets. J. Fuzzy Math. 2001, 9, 589-602.

6. Maji, P.K.; Biswas, R.; Roy, A.R. Intuitionistic fuzzy soft sets. J. Fuzzy Math. 2001, 9, 677-692.

7. Ali, M.I. A note on soft sets, rough soft sets and fuzzy soft sets. Appl. Soft Comput. 2011, 11, 3329-3332.

8. Yang, X.B.; Lin, T.Y.; Yang, J.Y.; Li, Y.; Yu, D.Y. Combination of interval-valued fuzzy set and soft set. Comput. Math. Appl. 2009, 58, 521-527. [CrossRef]

9. Jiang, Y.; Tang, Y.; Chen, Q.; Liu, H.; Tang, J. Interval-valued intuitionistic fuzzy soft sets and their properties. Comput. Math. Appl. 2010, 60, 906-918. [CrossRef]

10. Cuong, B.C. Picture fuzzy sets. J. Comput. Sci. Cybern. 2014, 30, 409-420.

11. Yang, Y.; Liang, C.; Ji, S.; Liu, T. Adjustable soft discernibility matrix based on picture fuzzy soft sets and its application in decision making. J. Int. Fuzzy Syst. 2015, 29, 1711-1722. [CrossRef]

12. Khan, M.J.; Kumam, P.; Ashraf, S.; Kumam, W. Generalized Picture Fuzzy Soft Sets and Their Application in Decision Support Systems. Symmetry 2019, 11, 415. [CrossRef]

13. Khan, M.J.; Kumam, P.; Liu P.; Kumam, W.; Ashraf, S. A Novel Approach to Generalized Intuitionistic Fuzzy Soft Sets and Its Application in Decision Support System. Mathematics 2019, 7, 742. [CrossRef]

14. Khan, M.J.; Kumam, P.; Liu, P.; Kumam, W.; Rehman, H. An adjustable weighted soft discernibility matrix based on generalized picture fuzzy soft set and its applications in decision making. J. Int. Fuzzy Syst. 2020, 38, 2103-2118. [CrossRef] 
15. Khan, M.J.; Kumam, P.; Liu, P.; Kumam, W. Another view on generalized interval valued intuitionistic fuzzy soft set and its applications in decision support system. J. Int. Fuzzy Syst. 2019, 1-16. [CrossRef]

16. Khan, M.J.; Phiangsungnoen, S.; Rehman, H.; Kumam, W. Applications of Generalized Picture Fuzzy Soft Set in Concept Selection. Thai J. Math. 2020, 18, 296-314.

17. Hayat, K.; Ali, M.I.; Cao, B.Y.; Karaaslan, F.; Yang, X.P. Another View of Aggregation Operators on Group-Based Generalized Intuitionistic Fuzzy Soft Sets: Multi-Attribute Decision Making Methods. Symmetry 2018, 10, 753. [CrossRef]

18. Liu, F.; Aiwu, G.; Lukovac, V.; Vukić, M. A multicriteria model for the selection of the transport service provider: A single valued neutrosophic dematel multicriteria model. Decis. Mak. Appl. Manag. Eng. 2018, 1, 121-130. [CrossRef]

19. Si, A.; Das, S.; Kar, S. An approach to rank picture fuzzy numbers for decision making problems. Decis. Mak. Appl. Manag. Eng. 2019, 2, 54-64. [CrossRef]

20. Yager, R.R. Pythagorean fuzzy subsets. In Proceedings of the 2013 Joint IFSA World Congress and NAFIPS Annual Meeting (IFSA/NAFIPS), Edmonton, AB, Canada, 24-28 June 2013; pp. 57-61.

21. Yager, R.R. Pythagorean membership grades in multicriteria decision making. IEEE Trans. Fuzzy Syst. 2014, 22, 958-965. [CrossRef]

22. Zhang, X.L.; Xu, Z.S. Extension of TOPSIS to multiple criteria decision making with Pythagorean fuzzy sets. Int. J. Intell. Syst. 2014, 29, 1061-1078. [CrossRef]

23. Peng, X.; Yang, Y. Some results for Pythagorean fuzzy sets. Int. J. Intell. Syst. 2015, 30, 1133-1160. [CrossRef]

24. Reformat, M.; Yager, R.R. Suggesting recommendations using Pythagorean fuzzy sets illustrated using Netflix movie data. In Proceedings of the International Conference on Information Processing and Management of Uncertainty in Knowledge-Based Systems, Montpellier, France, 15-19 July 2014; Laurent, A., Strauss, O., Bouchon-Meunier, B., Yager, R.R., Eds.; Springer: Cham, Switzerland, 2014; Volume, 442, pp. 546-556.

25. Peng, X.; Yuan, H.; Yang, Y. Pythagorean Fuzzy Information Measures and Their Applications. Int. J. Intell. Syst. 2017, 32, 991-1029. [CrossRef]

26. Ashraf, S.; Abdullah, S.; Mahmood, T.; Ghani, F.; Mahmood, T. Spherical fuzzy sets and their applications in multi-attribute decision making problems. J. Intell. Fuzzy Syst. 2019, 36, 2829-2844. [CrossRef]

27. Ashraf, S.; Abdullah, S.; Mahmood, T. Spherical fuzzy Dombi aggregation operators and their application in group decision making problems. J. Ambient Intell. Hum. Comput. 2019, 1-19. [CrossRef]

28. Ashraf, S.; Abdullah, S.; Aslam, M.; Qiyas, M.; Kutbi, M.A. Spherical fuzzy sets and its representation of spherical fuzzy t-norms and t-conorms. J. Intell. Fuzzy Syst. 2019, 36, 6089-6102. [CrossRef]

29. Rafiq, M.; Ashraf, S.; Abdullah, S.; Mahmood, T.; Muhammad, S. The cosine similarity measures of spherical fuzzy sets and their applications in decision making. J. Intell. Fuzzy Syst. 2019. [CrossRef]

30. Ashraf, S.; Abdullah, S.; Aslam, M. Symmetric sum based aggregation operators for spherical fuzzy information: Application in multi-attribute group decision making problem. J. Intell. Fuzzy Syst. 2020. [CrossRef]

31. Ngan, R.T.; Son, L.H.; Cuong, B.C.; Ali, M. H-max distance measure of intuitionistic fuzzy sets indecision making. Appl. Soft Comput. 2018, 69, 393-425. [CrossRef]

32. Garg, H.; Kumar, K. An advanced study on the similarity measures of intuitionistic fuzzy sets based on the set pair analysis theory and their application in decision making. Soft Comput. 2018. . [CrossRef]

33. Jiang, Q.; Jin, X.; Lee, S.; Yao, S. A new similarity/distance measure between intuitionistic fuzzy sets based on the transformed isosceles triangles and its applications to pattern recognition. Expert Syst. Appl. 2019, 116, 439-453. [CrossRef]

34. Stanujkic, D.; Karabasevic, D. An extension of the WASPAS method for decision-making problems with intuitionistic fuzzy numbers: A case of website evaluation. Oper. Res. Eng. Sci. Theory Appl. 2018, 1, $29-39$. [CrossRef]

(C) 2020 by the authors. Licensee MDPI, Basel, Switzerland. This article is an open access article distributed under the terms and conditions of the Creative Commons Attribution (CC BY) license (http://creativecommons.org/licenses/by/4.0/). 